\title{
One-loop impact factor for the emission of two gluons
}

\author{
Marc Canay ${ }^{a, b}$ and Vittorio Del Duca ${ }^{a, 1}$ \\ ${ }^{a}$ Institute for Theoretical Physics, ETH Zürich, \\ 8093 Zürich, Switzerland \\ ${ }^{b}$ Institut de Physique Théorique, CEA, CNRS, \\ Université Paris-Saclay, F-91191 Gif-sur-Yvette cedex, France \\ E-mail: marc.canay@ipht.fr, delducav@itp.phys.ethz.ch
}

ABSTRACT: We consider one-loop five-point QCD amplitudes in next-to-multi-Regge kinematics, and evaluate the one-loop impact factor for the emission of two gluons. This is the last ingredient which is necessary to evaluate the gluon-jet impact factor at NNLO accuracy in $\alpha_{s}$. It is also the first instance in which loop-level QCD amplitudes are evaluated in next-to-multi-Regge kinematics, which requires to apply a different Reggeisation ansatz to each colour-ordered amplitude.

Keywords: NLO Computations, QCD Phenomenology

ARXiv EPrint: 2103.16593

\footnotetext{
${ }^{1}$ On leave from: INFN, Laboratori Nazionali di Frascati, Italy.
} 


\section{Contents}

1 Introduction $\quad 1$

1.1 The Regge limit at leading logarithmic accuracy 3

1.2 The Regge limit at next-to-leading logarithmic accuracy 5

1.3 Moving toward next-to-next-to-leading logarithmic accuracy $\quad 7$

1.4 Plan 8

2 The impact factor for the emission of two gluons $\quad 8$

$\begin{array}{ll}2.1 & \text { The one-loop five-gluon amplitude in the next-to-multi-Regge kinematics } \quad 10\end{array}$

$\begin{array}{ll}2.2 & \text { One-loop impact factor for the emission of two gluons } \\ \end{array}$

$\begin{array}{lll}2.3 & \text { One-loop two-quark three-gluon amplitude in NMRK } & 14\end{array}$

$\begin{array}{lll}2.3 .1 & \text { The } p=2 \text { term } & 17\end{array}$

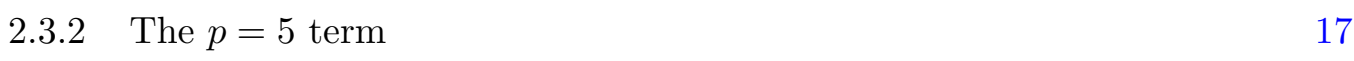

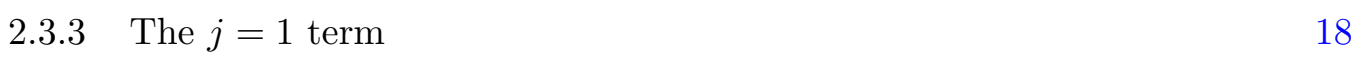

$\begin{array}{ll}2.3 .4 \text { Regge factorisation } & 19\end{array}$

$\begin{array}{ll}2.4 \text { Helicity-violating contributions } & 19\end{array}$

3 Discussion and conclusions $\quad 20$

$\begin{array}{ll}\text { A Multiparton kinematics } & 21\end{array}$

B Multi-Regge kinematics $\quad 22$

C Next-to-multi-Regge kinematics $\quad 23$

$\begin{array}{ll}\text { D Anomalous dimensions } & 24\end{array}$

E One-loop five-gluon colour-ordered amplitudes $\quad 24$

$\begin{array}{lll}\text { F The one-loop five-gluon amplitude in NMRK } & 27\end{array}$

G Coefficients of the one-loop two-quark three-gluon amplitude $\begin{array}{ll}\text { in NMRK } & 28\end{array}$

\section{Introduction}

The Regge limit of $2 \rightarrow 2$ scattering amplitudes of massless gauge theories, like Yang-Mills theories or $\mathcal{N}=4$ Super Yang-Mills (SYM), has been studied since the pioneering work of Lipatov on the gluon Reggeisation [1], and the more so in the last decade, after realising that amplitudes in the Regge limit are endowed with a rich mathematical structure [2-7]. 


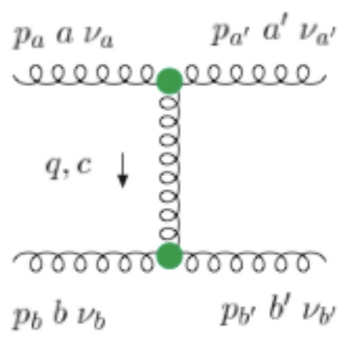

Figure 1. Amplitude for $g g \rightarrow g g$ scattering in the Regge limit. The green blobs represent the impact factors.

In the Regge limit, in which the squared centre-of-mass energy $s$ is much larger than the momentum transfer $t, s \gg|t|, 2 \rightarrow 2$ scattering amplitudes are dominated by gluon exchange in the $t$ channel. Contributions which do not feature gluon exchange in the $t$ channel are power suppressed in $t / s$. In particular, at tree level we can write the $2 \rightarrow 2$ amplitudes in a factorised way. For example, the tree amplitude for gluon-gluon scattering $g_{a} g_{b} \rightarrow g_{a^{\prime}} g_{b^{\prime}}$ at fixed helicities may be written as $[1,8]$,

$$
\mathcal{M}_{g g \rightarrow g g}^{(0)}=\left[g_{S}\left(F^{a^{\prime}}\right)_{a c} C^{g(0)}\left(p_{a}^{\nu_{a}} ; p_{a^{\prime}}^{\nu_{a^{\prime}}}\right)\right] \frac{s}{t}\left[g_{S}\left(F^{b^{\prime}}\right)_{c b} C^{g(0)}\left(p_{b}^{\nu_{b}} ; p_{b^{\prime}}^{\nu_{b^{\prime}}}\right)\right]
$$

with $s=\left(p_{a}+p_{b}\right)^{2}, q=p_{b^{\prime}}+p_{b}$ and $t=q^{2} \simeq-\left|q_{\perp}\right|^{2}$, figure 1 , and $\left(F^{c}\right)_{a b}=i \sqrt{2} f^{a c b}$, and where the superscripts $\nu$ label the helicities. ${ }^{1}$ As it is apparent from the colour coefficient $\left(F^{a^{\prime}}\right)_{a c}\left(F^{b^{\prime}}\right)_{c b}$, in eq. (1.1) only the antisymmetric octet $\mathbf{8}_{a}$ is exchanged in the $t$ channel. Finally, eq. (1.1) yields the four-gluon amplitude in terms of one colour-ordered amplitude, in agreement with the Bern-Carrasco-Johansson (BCJ) relations [9], which fix the number of colour-ordered amplitudes at $(n-3)$ !.

Since the four-gluon amplitude is a maximally helicity-violating (MHV) amplitude, eq. (1.1) describes $\left(\begin{array}{l}4 \\ 2\end{array}\right)=6$ helicity configurations. However, at leading power in $t / s$, helicity is conserved along the $s$-channel direction in Minkowski space, so in eq. (1.1) four helicity configurations are leading, two for each tree impact factor, $g^{*} g \rightarrow g$, with $g^{*}$ an off-shell gluon [10], ${ }^{2}$

$$
C^{g(0)}\left(p_{a}^{-} ; p_{a^{\prime}}^{+}\right)=1, \quad C^{g(0)}\left(p_{b}^{-} ; p_{b^{\prime}}^{+}\right)=-\frac{p_{b^{\prime} \perp}^{*}}{p_{b^{\prime} \perp}}
$$

The impact factors transform under parity into their complex conjugates,

$$
\left[C^{g}\left(p^{\nu} ; p^{\nu^{\prime}}\right)\right]^{*}=C^{g}\left(p^{-\nu} ; p^{-\nu^{\prime}}\right)
$$

The helicity-flip impact factor $C^{g(0)}\left(p^{+} ; p^{+}\right)$and its parity conjugate $C^{g(0)}\left(p^{-} ; p^{\prime-}\right)$ are power suppressed in $t / s$.

\footnotetext{
${ }^{1}$ We take all the momenta as outgoing, so the helicities for incoming partons are reversed, see appendix A.

${ }^{2}$ In the literature, the colour coefficient of the gluon-gluon scattering amplitude in the Regge limit is usually written as $f^{a a^{\prime} c} f^{b b^{\prime} c}$. In eq. (1.1), we follow the cyclic ordering of the gluons in the amplitude, obtaining a relative minus sign, which is absorbed into the definition of $C^{g(0)}\left(p_{b}^{-} ; p_{b^{\prime}}^{+}\right)$in eq. (1.2).
} 
The tree amplitudes for quark-gluon or quark-quark scattering have the same form as eq. (1.1), up to replacing one or both gluon impact factors $C^{g(0)}(1.2)$ with quark impact factors $C^{q(0)}$, and the colour factors $\left(F^{c}\right)_{a b}$ in the adjoint representation with the colour factors $T_{i j}^{c}$ in the fundamental representation of SU(3), normalised as $\operatorname{Tr}\left(T^{a} T^{b}\right)=T_{F} \delta^{a b}$, with $T_{F}=1$. So in the Regge limit, the $2 \rightarrow 2$ scattering amplitudes factorise into gluon or quark impact factors and a gluon propagator in the $t$ channel, and are uniquely determined by them.

The loop corrections to an amplitude introduce poles and branch cuts, which are dictated by the analytic structure and constrained by the symmetries of the amplitude. In particular, in the Regge limit $s \simeq-u \gg-t, 2 \rightarrow 2$ scattering amplitudes are symmetric under $s \leftrightarrow u$ crossing, thus we may consider amplitude states whose kinematic and colour coefficients have a definite signature under $s \leftrightarrow u$ crossing,

$$
\mathcal{M}_{4}^{( \pm)}(s, t)=\frac{\mathcal{M}_{4}(s, t) \pm \mathcal{M}_{4}(u, t)}{2}
$$

with $u=-s-t \simeq-s$, such that $\mathcal{M}_{4}^{(-)}(s, t)\left(\mathcal{M}_{4}^{(+)}(s, t)\right)$ has kinematic and colour coefficients which are both odd (even) under $s \leftrightarrow u$ crossing. Further, higher-order contributions to $g g \rightarrow g g$ scattering in general involve additional colour structures, as dictated by the decomposition of the product $\boldsymbol{8}_{a} \otimes \mathbf{8}_{a}$ into irreducible representations,

$$
\mathbf{8}_{a} \otimes \mathbf{8}_{a}=\left\{\mathbf{1} \oplus \mathbf{8}_{s} \oplus \mathbf{2 7}\right\} \oplus\left[\mathbf{8}_{a} \oplus \mathbf{1 0} \oplus \overline{\mathbf{1 0}}\right],
$$

where in curly (square) brackets are the representations which are even (odd) under $s \leftrightarrow u$ crossing.

\subsection{The Regge limit at leading logarithmic accuracy}

When loop corrections to the tree amplitude (1.1) are considered, it is found that at leading logarithmic (LL) accuracy in $\log (s /|t|)$, the four-gluon amplitude is given to all orders in $\alpha_{s}$ by $[1,8]$

$$
\left.\mathcal{M}_{g g \rightarrow g g}\right|_{L L}=\left[g_{S}\left(F^{a^{\prime}}\right)_{a c} C^{g(0)}\left(p_{a}^{\nu_{a}} ; p_{a^{\prime}}^{\nu_{a^{\prime}}}\right)\right] \frac{s}{t}\left(\frac{s}{\tau}\right)^{\alpha(t)}\left[g_{S}\left(F^{b^{\prime}}\right)_{c b} C^{g(0)}\left(p_{b}^{\nu_{b}} ; p_{b^{\prime}}^{\nu_{b^{\prime}}}\right)\right]
$$

with $\alpha(t)$ related to the loop transverse-momentum integration, and where $\tau>0$ is a Regge factorisation scale. $\tau$ is of order of $t$, and much smaller than $s$, however the precise definition of $\tau$ is immaterial to LL accuracy, where one can suitably fix $\tau=-t$. Regulating $\alpha(t)$ in $d=4-2 \epsilon$ dimensions, one obtains

$$
\alpha(t) \equiv \frac{g_{S}^{2}}{(4 \pi)^{2}} \alpha^{(1)}(t)=\frac{\alpha_{S}}{4 \pi} N_{c} \frac{2}{\epsilon}\left(\frac{\mu^{2}}{-t}\right)^{\epsilon} \kappa_{\Gamma}
$$

with $N_{c}$ the number of colours, $\alpha_{S}=g_{S}^{2} /(4 \pi)$ and

$$
\kappa_{\Gamma}=(4 \pi)^{\epsilon} \frac{\Gamma(1+\epsilon) \Gamma^{2}(1-\epsilon)}{\Gamma(1-2 \epsilon)} .
$$



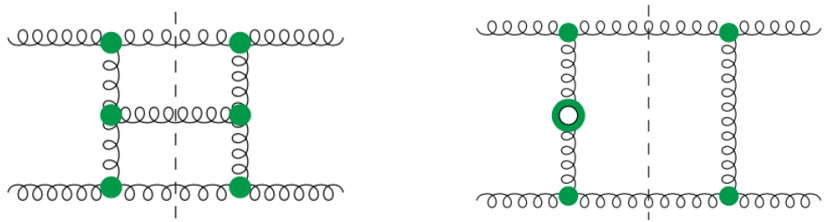

Figure 2. (a) Tree five-gluon amplitude. The green blob along the gluon ladder represents the central-emission vertex. (b) Regge trajectory in the one-loop four-gluon amplitude. The pierced blob represents the one-loop Regge trajectory.

The prominent facts of eq. (1.6) are that at LL accuracy the amplitude (1.6) is still real, the one-loop result (1.7) exponentiates, and the antisymmetric octet $\boldsymbol{8}_{a}$ is still the only colour representation exchanged in the $t$ channel. Thus, at LL accuracy,

$$
\left.\mathcal{M}_{g g \rightarrow g g}\right|_{L L}=\left.\mathcal{M}_{g g \rightarrow g g}^{(-)\left[8_{a}\right]}\right|_{L L},\left.\quad \quad \mathcal{M}_{g g \rightarrow g g}^{(+)}\right|_{L L}=0
$$

$\alpha(t)$ is called Regge trajectory, and the exponentiation of $\log (s /|t|)$ in the one-loop result (1.7) is called gluon Reggeisation [11]. Because of eq. (1.6), factorisation holds at LL accuracy just like at tree level, i.e. the amplitudes for quark-gluon or quark-quark scattering at LL accuracy have the same form as eq. (1.6), up to replacing one or both colour and impact factors for gluons with the ones for quarks.

The gluon Reggeisation at LL accuracy constitutes the backbone of the Balitsky-FadinKuraev-Lipatov (BFKL) equation [8, 12-14], which at $t=0$ describes the $s$-channel cut forward amplitude, which through the optical theorem is equivalent to the squared amplitude integrated over all the allowed final states. The BFKL equation sums the terms of $\mathcal{O}\left(\alpha_{S}^{n} \log ^{n}(s /|t|)\right)$ and describes the evolution of the gluon ladder in transverse momentum space, after the gluon rapidities have been integrated out. Since in the BFKL equation also the real emissions are included, in order to match the LL accuracy of the virtual corrections (1.6), amplitudes with five or more gluons are taken in the multi-Regge kinematics (MRK), which requires that the gluons are strongly ordered in rapidity and have comparable transverse momentum, see appendix B. The MRK rationale is that each gluon emitted along the ladder requires a factor of $\alpha_{S}$ and after being integrated out in rapidity yields a factor of $\log (s /|t|)$, in such a way that each gluon emitted along the ladder contributes a factor of $\mathcal{O}\left(\alpha_{S} \log (s /|t|)\right)$. Five-gluon amplitudes in MRK allow one to compute the emission of a gluon along the gluon ladder. The factor associated to that gluon emission is called central-emission vertex, or Lipatov vertex [1]. The central-emission vertex constitutes the kernel of the BFKL equation. Beyond five gluons, amplitudes in MRK assume an iterative structure, with a central-emission vertex for each gluon emitted along the ladder. Thus, the building blocks of the BFKL equation are the central-emission vertex at tree level, figure 2(a), and the Regge trajectory at one loop (1.7), figure 2(b). Finally, the impact factors (1.2) are not part of the gluon ladder, but sit at its ends, and when they are squared they constitute the jet impact factors and contribute to any jet cross section [15-21] which is computed through the BFKL equation. 

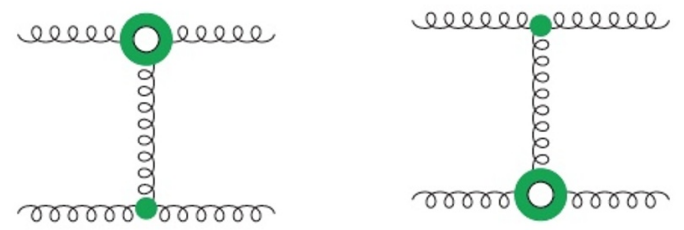

Figure 3. Impact factors in the one-loop four-gluon amplitude. The pierced blobs represent the one-loop impact factors $C^{g(1)}(t)$.

\subsection{The Regge limit at next-to-leading logarithmic accuracy}

At next-to-leading-logarithmic (NLL) accuracy, keeping the $s \leftrightarrow u$ crossing symmetry (1.4) into account, the exchange of one Reggeised gluon of eq. (1.6) generalises to [22]

$$
\mathcal{M}_{g g \rightarrow g g}^{(-)\left[8_{a}\right]}=\frac{1}{2}\left[g_{S}\left(F^{a^{\prime}}\right)_{a c} C^{g}\left(p_{a}^{\nu_{a}} ; p_{a^{\prime}}^{\nu_{a^{\prime}}}\right)\right] \frac{s}{t}\left[\left(\frac{s}{\tau}\right)^{\alpha(t)}+\left(\frac{-s}{\tau}\right)^{\alpha(t)}\right]\left[g_{S}\left(F^{b^{\prime}}\right)_{c b} C^{g}\left(p_{b}^{\nu_{b}} ; p_{b^{\prime}}^{\nu_{b^{\prime}}}\right)\right],
$$

where the colour and kinematic parts of the amplitude are each odd under $s \leftrightarrow u$ crossing. Further, by expanding the gluon Regge trajectory,

$$
\alpha(t)=\frac{\alpha_{S}}{4 \pi} \alpha^{(1)}(t)+\left(\frac{\alpha_{S}}{4 \pi}\right)^{2} \alpha^{(2)}(t)+\mathcal{O}\left(\alpha_{S}^{3}\right),
$$

with $\alpha^{(1)}(t)$ given in eq. (1.7), and the helicity-conserving impact factor,

$$
C^{g}\left(p_{j}^{\mp} ; p_{j^{\prime}}^{ \pm}\right)=C^{g(0)}\left(p_{j}^{\mp} ; p_{j^{\prime}}^{ \pm}\right)\left(1+\frac{\alpha_{S}}{4 \pi} C_{-+}^{g(1)}(t, \tau)+\mathcal{O}\left(\alpha_{S}^{2}\right)\right),
$$

with $j=a, b$, see figure 3, one can write the amplitude (1.10) as a double expansion in the strong coupling $\alpha_{S}$ and in $\log (s / \tau)$,

$$
\mathcal{M}_{g g \rightarrow g g}^{(-)[8 a]}=\mathcal{M}_{g g \rightarrow g g}^{(0)}\left(1+\sum_{\ell=1}^{\infty}\left(\frac{\alpha_{S}}{4 \pi}\right)^{\ell} M_{g g \rightarrow g g}^{(\ell)\left[8_{a}\right]}\right),
$$

with

$$
M_{g g \rightarrow g g}^{(\ell)\left[8_{a}\right]}=\sum_{i=0}^{\ell} M_{g g \rightarrow g g}^{(\ell, i)\left[8_{a}\right]} \log ^{i}\left(\frac{s}{\tau}\right),
$$

where the $M_{g g \rightarrow g g}^{(\ell, \ell)[8 a}$ coefficients have LL accuracy, the $M_{g g \rightarrow g g}^{(\ell, \ell-1)\left[8_{a}\right]}$ coefficients have NLL accuracy, and in general the $M_{g g \rightarrow g g}^{(\ell, \ell-k)\left[8_{a}\right]}$ coefficients have $\mathrm{N}^{\mathrm{k}} \mathrm{LL}$ accuracy.

Beyond LL accuracy, in the gluon ladder the exchange of two or more Reggeised gluons may appear. Further, all the colour representations (1.5) exchanged in the $t$ channel may contribute. However, at NLL accuracy, the real part of the amplitude is entirely given by the antisymmetric octet $\boldsymbol{8}_{a}$ through eq. (1.10),

$$
\operatorname{Re}\left[\mathcal{M}_{g g \rightarrow g g}\right]_{\mathrm{NLL}}=\operatorname{Re}\left[\mathcal{M}_{g g \rightarrow g g}^{(-)\left[8_{a}\right]}\right],
$$

which, once eq. (1.10) is expanded at one and two loops reads,

$$
\begin{aligned}
\operatorname{Re}\left[\mathcal{M}_{g g \rightarrow g g}^{(1)}\right]_{\mathrm{NLL}} & =\alpha^{(1)}(t) \log \left(\frac{s}{\tau}\right)+2 \operatorname{Re}\left[C^{g(1)}(t, \tau)\right], \\
\operatorname{Re}\left[\mathcal{M}_{g g \rightarrow g g}^{(2)}\right]_{\mathrm{NLL}} & =\frac{1}{2}\left(\alpha^{(1)}(t)\right)^{2} \log ^{2}\left(\frac{s}{\tau}\right)+\left(\alpha^{(2)}(t)+2 \operatorname{Re}\left[C^{g(1)}(t, \tau)\right] \alpha^{(1)}(t)\right) \log \left(\frac{s}{\tau}\right) .
\end{aligned}
$$



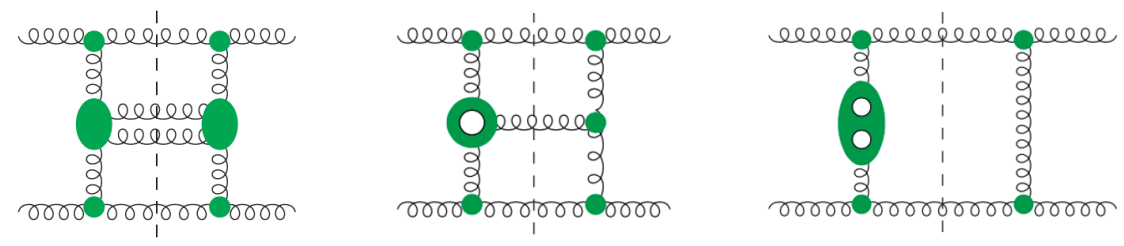

Figure 4. (a) Tree six-gluon amplitude. The oval blob represents the central-emission vertex - the emission of two gluons or of a quark-antiquark pair along the gluon ladder. (b) One-loop five-gluon amplitude. The pierced blob represents the one-loop corrections to the central-emission vertex. (c) Regge trajectory in the two-loop four-gluon amplitude. The double pierced oval represents the two-loop Regge trajectory.

Beyond two loops, no more coefficients occur at NLL accuracy, i.e. the gluon-gluon scattering amplitude is uniquely determined by eq. (1.10), in terms of the two-loop Regge trajectory $\alpha^{(2)}(t)$ [23-27] and the one-loop impact factor $C^{(1)}$ [22, 28-31]. Accordingly, gluon Reggeisation is extended to NLL accuracy [32, 33]. Further, because of eq. (1.10) factorisation still holds, so the amplitudes for quark-gluon or quark-quark scattering have the same form as eq. (1.10), up to replacing one or both colour and impact factors for gluons with the ones for quarks.

Likewise, the BFKL equation is extended to NLL accuracy [34, 35], which sums the terms of $\mathcal{O}\left(\alpha_{S}^{n} \log ^{n-1}(s / \tau)\right)$. At NLL accuracy, the kernel of the BFKL equation is given by the radiative corrections to the central-emission vertex, i.e. the emission of two gluons or of a quark-antiquark pair along the gluon ladder [36-40], figure 4(a), and the one-loop corrections to the central-emission vertex [22, 31, 41-43], figure 4(b). The infrared divergences of the radiative corrections to the central-emission vertex cancel the divergences of the twoloop Regge trajectory, figure 4(c). The emission of two gluons or of a quark-antiquark pair along the gluon ladder requires the adoption of a next-to-multi-Regge kinematics (NMRK), in which the gluons are strongly ordered in rapidity except for two gluons or for a quarkantiquark pair emitted along the gluon ladder. The NMRK rationale is that when the two gluons or the quark-antiquark pair are integrated out in rapidity, they yield a factor of $\mathcal{O}\left(\alpha_{S}^{2} \log (s / \tau)\right)$, thus contributing to NLL accuracy. Finally, in order to compute jet cross sections at NLL accuracy through the BFKL equation [44-46], one needs to compute the jet impact factors at next-to-leading order (NLO) in $\alpha_{S}[47,48]$. To that effect, one needs the one-loop impact factor $C^{(1)}$ and the impact factor for the emission of two gluons or of a quark-antiquark pair [36-40], evaluated in NMRK, section C, in which the gluons are strongly ordered in rapidity except for two partons emitted at one end of the ladder.

At NLL accuracy, in addition to the imaginary part of the antisymmetric octet $\mathbf{8}_{a}$ in eq. (1.10), ${ }^{3}$ all the colour representations which are even under $s \leftrightarrow u$ crossing (1.5),

\footnotetext{
${ }^{3}$ In order to avoid an imaginary part in the antisymmetric octet $\mathbf{8}_{a}$, one can define the combination of logarithms [49],

$$
L=\frac{1}{2}\left(\log \left(\frac{s}{\tau}\right)+\log \left(\frac{u}{\tau}\right)\right)=\log \left(\frac{s}{\tau}\right)-i \frac{\pi}{2} .
$$

Using $L$ as the resummation parameter, one may identify odd(even)-signature states with the real (imaginary) part of the amplitude, and thus with the exchange of an odd (even) number of Reggeised gluons, which is a convenient bookkeping device to classify the various contributions to the $t$-channel exchange. At the accuracy of our study, this is immaterial, and we will keep using $\log (s / \tau)$ as the resummation parameter.
} 
i.e. the singlet $\mathbf{1}$, the symmetric octet $\mathbf{8}_{s}$ and the $\mathbf{2 7}$, appear in the imaginary part of the gluon-gluon scattering amplitude, already in the non-logarithmic term of the one-loop amplitude $M_{g g \rightarrow g g}^{(1,0)}$ [30]. Just like the BFKL equation [8, 13, 14], which is described by the exchange of two Reggeised gluons in the $t$ channel and displays a cut in the $s$ channel, the imaginary part of the gluon-gluon amplitude features a cut and is described by the exchange of two Reggeised gluons [50, 51], and just like the BFKL equation [3] its finite parts can be expressed in terms of single-valued harmonic polylogarithms [7]. The analyses of refs. $[7,51]$ complete the study of gluon-gluon scattering amplitudes at NLL accuracy. We note that the imaginary part of the gluon-gluon amplitude at NLL accuracy can only contribute to a squared amplitude, and thus to a cross section, at next-to-next-to-leadinglogarithmic (NNLL) accuracy.

\subsection{Moving toward next-to-next-to-leading logarithmic accuracy}

At NNLL accuracy, both the antisymmetric octet $\mathbf{8}_{a}$ and the $\mathbf{1 0} \oplus \overline{\mathbf{1 0}}$ feature a cut in the $s$ channel, which is described by the exchange of three Reggeised gluons in the $t$ channel $[49,52,53]$. This is in addition to the exchange of one Reggeised gluon, which is still given by eq. (1.10). Thus, Regge pole factorisation, which is based on the exchange of one Reggeised gluon, does not hold any more [27]. Accordingly, in the non-logarithmic term of the two-loop amplitude, $M_{g g \rightarrow g g}^{(2,0)}$, both the two-loop impact factor and the threeReggeised-gluon exchange ${ }^{4}$ contribute.

However, in $M_{g g \rightarrow g g}^{(2,0)}$, the factorisation-violating term is a $1 / \varepsilon^{2}$ pole, whose coefficient is subleading in $N_{c}$ [27]. Since it is an infrared pole, it can be analysed through infrared factorisation, which shows that, in addition to the usual diagonal terms of the colour octet exchange, also non-diagonal terms in the $t$-channel colour basis contribute to the real part of $M_{g g \rightarrow g g}^{(2,0)}[55,56]$. Then one can disentangle the infrared poles of the two-loop impact factor (1.10) from the ones of factorisation-violating terms by identifying the impact factor with the diagonal terms and the factorisation-violating terms with the off-diagonal ones. Carrying this prescription on to the next loop order, one can predict how the infrared poles of the factorisation-violating term propagate into the single-logarithmic term of the threeloop amplitude $M_{g g \rightarrow g g}^{(3,1)}[57,58]$, and thus have a prescription to disentangle the infrared poles of the three-loop Regge trajectory, which should be given by the cusp anomalous dimension [59], from the ones of the factorisation-violating terms. The disentanglement, which is based on such a prescription, has been confirmed by the explicit computation of the three-loop four-point function of $\mathcal{N}=4 \mathrm{SYM}$ [60]. We stress, though, that the disentanglement above is valid only for the infrared poles through three loops. In order to predict factorisation-violating terms beyond three loops and including the finite terms, one must be able to compute the three Reggeised-gluon exchange [7, 49].

The possibility of disentangling terms which are based on the exchange of one Reggeised gluon from factorisation-violating terms makes us hope that the BFKL equation, which is based on the exchange of one Reggeised gluon, could be extended to NNLL accuracy. Further, there are reasons, which are based on the integrability of amplitudes in MRK in

\footnotetext{
${ }^{4}$ At NNLL accuracy, the three-Reggeised-gluon exchange is currently known up to four loops [54].
} 
the large $N_{c}$ limit [61] and on the colour structure of the corrections found through four loops in ref. [54], to believe that the Regge pole factorisation would be simpler in the large $N_{c}$ limit. This warrants an analysis of the terms which would contribute to the BFKL equation at NNLL accuracy.

At NNLL accuracy, the kernel of the BFKL equation will be given by the emission of three partons along the gluon ladder [62-64], evaluated in next-to-next-to-multi-Regge kinematics (NNMRK); by the one-loop corrections to the emission of two gluons or of a quark-antiquark pair along the gluon ladder, evaluated in NMRK; and by the twoloop corrections to the central-emission vertex. The last two contributions are yet to be determined. The infrared divergences of the contributions outlined above will cancel the divergences of the three-loop Regge trajectory, which is so far only known in a specific scheme [49].

Finally, in order to compute jet cross sections at NNLL accuracy through the BFKL equation, one needs to compute the jet impact factors at next-to-next-to-leading order (NNLO) in $\alpha_{S}$. To that effect, one needs the tree impact factor for the emission of three partons at one end of the ladder [62], evaluated in NNMRK; the two-loop impact factor $C^{(2)}$ [58], and the one-loop impact factor for the emission of two gluons or of a quarkantiquark pair, evaluated in NMRK. Further, one must include the square of the one-loop helicity-violating impact factor, which is recalled in eqs. (2.51) and (2.52).

\subsection{Plan}

Using the one-loop corrections to the five-gluon amplitudes [65] and to the two-quark threegluon amplitudes [66], in this paper we evaluate the one-loop impact factor for the emission of two gluons. This is the last ingredient which is necessary to evaluate the gluon-jet impact factor at NNLO accuracy in $\alpha_{S}$.

Section 2 is devoted to the computation of the one-loop impact factor for the emission of two gluons. Firstly, we recall the tree-level impact factor; then in section 2.1, we consider the one-loop five-gluon amplitude in NMRK, and apply the Reggeisation ansatz to each colour-ordered amplitude (2.13); from that we extract in section 2.2 the one-loop impact factor for the emission of two gluons (2.24). These are the main results of the paper. Then, in order to verify that Regge factorisation holds at NLL accuracy in NMRK, we consider in section 2.3 the one-loop two-quark three-gluon amplitude in NMRK, and we extract again the one-loop impact factor for the emission of two gluons, finding agreement with the previous computation. Finally, in section 2.4 we compute the one-loop helicityviolating impact factor. In section 3, we draw our conclusions. Several appendices are given, which contain information about the kinematic approximations, and the one-loop five-point amplitudes in general kinematics and in NMRK.

\section{The impact factor for the emission of two gluons}

We consider the production of three gluons of momenta $p_{1}, p_{2}$ and $p_{b^{\prime}}$ in the scattering of two gluons of momenta $p_{a}$ and $p_{b}$. We suppose that the outgoing gluons are in NMRK, 


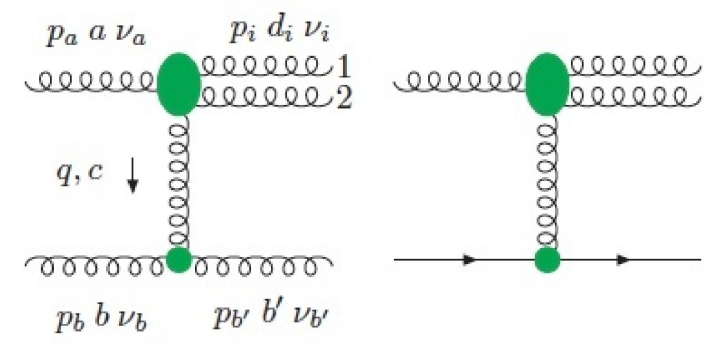

Figure 5. Tree $(a)$ five-gluon amplitude for the $g_{a} g_{b} \rightarrow g_{1} g_{2} g_{b^{\prime}}$ scattering, and $(b)$ two-quark three-gluon amplitude for the $g_{a} q_{b} \rightarrow g_{1} g_{2} q_{b^{\prime}}$ scattering, in the NMRK of eq. (2.1). The oval blob represents the impact factor for the emission of two gluons.

section $\mathrm{C}$, with rapidities and transverse momenta,

$$
y_{1} \simeq y_{2} \gg y_{b^{\prime}} ; \quad\left|p_{1 \perp}\right| \simeq\left|p_{2 \perp}\right| \simeq\left|p_{b^{\prime} \perp}\right| .
$$

In the NMRK region of eq. (2.1), the tree amplitude for the $g_{a} g_{b} \rightarrow g_{1} g_{2} g_{b^{\prime}}$ scattering is $[40]$

$$
\left.\mathcal{M}_{g g \rightarrow g g g}^{(0)}\right|_{\mathrm{NMRK}}=\left.\sum_{\sigma \in S_{2}} g_{S}^{3}\left(F^{d_{\sigma_{1}}} F^{d_{\sigma_{2}}}\right)_{a c}\left(F^{b^{\prime}}\right)_{c b} m_{5 g}^{(0)}\left(p_{a}^{\nu_{a}}, p_{\sigma_{1}}^{\nu_{\sigma_{1}}}, p_{\sigma_{2}}^{\nu_{\sigma_{2}}}, p_{b^{\prime}}^{\nu_{b^{\prime}}}, p_{b}^{\nu_{b}}\right)\right|_{\mathrm{NMRK}}
$$

where the sum is over the permutations of the labels 1 and 2 , and the colour-ordered amplitude is

$$
\left.m_{5 g}^{(0)}\left(p_{a}^{\nu_{a}}, p_{1}^{\nu_{1}}, p_{2}^{\nu_{2}}, p_{b^{\prime}}^{\nu_{b^{\prime}}}, p_{b}^{\nu_{b}}\right)\right|_{\mathrm{NMRK}}=A^{g g(0)}\left(p_{a}^{\nu_{a}} ; p_{1}^{\nu_{1}}, p_{2}^{\nu_{2}}\right) \frac{s}{t} C^{g(0)}\left(p_{b}^{\nu_{b}} ; p_{b^{\prime}}^{\nu_{b^{\prime}}}\right),
$$

with $s=p_{a}+p_{b}, q=p_{b^{\prime}}+p_{b}$ and $t \simeq-\left|q_{\perp}\right|^{2}$, figure 5(a). Eq. (2.2) yields the five-gluon amplitude in terms of two colour-ordered amplitudes, again in agreement with the BernCarrasco-Johansson (BCJ) counting of $(n-3)$ ! independent colour-ordered amplitudes.

In eq. (2.3), the impact factor $A^{g g(0)}\left(p_{a}^{\nu_{a}} ; p_{1}^{\nu_{1}}, p_{2}^{\nu_{2}}\right)$ for $g^{*} g \rightarrow g g$ can be written as

$$
A^{g g(0)}\left(p_{a}^{\nu_{a}} ; p_{1}^{\nu_{1}}, p_{2}^{\nu_{2}}\right)=D^{g g}\left(p_{a}^{\nu_{a}} ; p_{1}^{\nu_{1}}, p_{2}^{\nu_{2}}\right) B^{\tilde{\nu}}\left(p_{1}, p_{2}\right),
$$

with

$$
\begin{aligned}
& D^{g g}\left(p_{a}^{-} ; p_{1}^{+}, p_{2}^{+}\right)=1, \\
& D^{g g}\left(p_{a}^{+} ; p_{1}^{-}, p_{2}^{+}\right)=x_{1}^{2}, \\
& D^{g g}\left(p_{a}^{+} ; p_{1}^{+}, p_{2}^{-}\right)=x_{2}^{2},
\end{aligned}
$$

and

$$
\tilde{\nu}=\operatorname{sign}\left(\nu_{a}+\nu_{1}+\nu_{2}\right)
$$

and $^{5}$

$$
B^{+}\left(p_{1}, p_{2}\right)=\frac{p_{1 \perp}+p_{2 \perp}}{p_{1 \perp}} \sqrt{\frac{x_{1}}{x_{2}}} \frac{1}{\langle 12\rangle},
$$

\footnotetext{
${ }^{5}$ Note that since $\left(F^{c}\right)_{a b}=i \sqrt{2} f^{a c b}$, the coefficient (2.7) and the central-emission vertex (2.11) do not display the usual overall $\sqrt{2}$ factor.
} 
and where the momentum fractions are

$$
x_{i}=\frac{p_{i}^{+}}{p_{1}^{+}+p_{2}^{+}}, \quad i=1,2 .
$$

The $B$ coefficient (2.7) displays a collinear divergence as $p_{1} \cdot p_{2} \rightarrow 0$. The $D$ coefficients (2.5) are real, and so invariant under parity, while $B$ transforms under parity into its complex conjugate,

$$
B^{-}\left(p_{1}, p_{2}\right)=\left[B^{+}\left(p_{1}, p_{2}\right)\right]^{*} .
$$

The impact factor $A^{g g(0)}\left(p_{a}^{\nu_{a}} ; p_{2}^{\nu_{2}}, p_{1}^{\nu_{1}}\right)$ is obtained from eq. (2.4) by swapping the labels 1 and 2 .

Since the five-gluon amplitude is a MHV amplitude, eq. (2.2) describes $2\left(\begin{array}{c}5 \\ 2\end{array}\right)=20$ helicity configurations. However, at leading power in $t / s$, only 12 helicity configurations are leading. The impact factor (2.4) describes six helicity configurations, three out of which are given by eq. (2.5), and three more by parity conjugation. They are multiplied by the two given by the impact factor $C^{g(0)}\left(p_{b}^{\mp} ; p_{b^{\prime}}^{ \pm}\right)(1.2)$. Then there are eight helicity configurations which are power suppressed in $t / s$. Three of them are associated to $C^{g(0)}\left(p_{b}^{-} ; p_{b^{\prime}}^{-}\right)$, multiplied by the impact factor (2.4) with the helicity configurations (2.5), one is associated to $C^{g(0)}\left(p_{b}^{-} ; p_{b^{\prime}}^{-}\right)$multiplied by $A^{g g(0)}\left(p_{a}^{+} ; p_{1}^{+}, p_{2}^{+}\right)$. The remaining four are obtained from the four above by parity conjugation.

In MRK, appendix B, with $p_{1}^{+} \gg p_{2}^{+}$, the impact factor $A^{g g(0)}\left(p_{a}^{\nu_{a}} ; p_{2}^{\nu_{2}}, p_{1}^{\nu_{1}}\right)$ is power suppressed in $p_{2}^{+} / p_{1}^{+}$because of the $F$ coefficient $(2.7)$, while $A^{g g(0)}\left(p_{a}^{\nu_{a}} ; p_{1}^{\nu_{1}}, p_{2}^{\nu_{2}}\right)$ factorises as

$$
\lim _{p_{1}^{+} \gg p_{2}^{+}} A^{g g(0)}\left(p_{a}^{\nu_{a}} ; p_{1}^{\nu_{1}}, p_{2}^{\nu_{2}}\right)=C^{g(0)}\left(p_{a}^{\nu_{a}} ; p_{1}^{\nu_{1}}\right) \frac{1}{t_{1}} V^{g(0)}\left(q_{1}, p_{2}^{\nu_{2}}\right),
$$

where $q_{1}=-\left(p_{a}+p_{1}\right), q_{2}=q_{1}-p_{2}, t_{1}=q_{1}^{2} \simeq-q_{1 \perp} q_{1 \perp}^{*}$, and where $C^{g(0)}\left(p_{a}^{\nu_{a}} ; p_{1}^{\nu_{1}}\right)$ is given in eq. (1.2), while the tree central-emission vertex is

$$
V^{g(0)}\left(q_{1}, p_{2}^{+}\right)=\frac{q_{1 \perp}^{*} q_{2 \perp}}{p_{2 \perp}}
$$

Accordingly, the five-gluon amplitude (2.2) takes the factorised form,

$$
\begin{aligned}
\left.\mathcal{M}_{g g \rightarrow g g g}^{(0)}\right|_{\mathrm{MRK}}= & s\left[g_{S}\left(F^{d_{1}}\right)_{a c_{1}} C^{g(0)}\left(p_{a}^{\nu_{a}} ; p_{1}^{\nu_{1}}\right)\right] \frac{1}{t_{1}} \\
& \times\left[g_{S}\left(F^{d_{2}}\right)_{c_{1} c_{2}} V^{g(0)}\left(q_{1}, p_{2}^{\nu_{2}}\right)\right] \frac{1}{t_{2}}\left[g_{S}\left(F^{b^{\prime}}\right)_{c_{2} b} C^{g(0)}\left(p_{b}^{\nu_{b}} ; p_{b^{\prime}}^{\nu_{\prime^{\prime}}}\right)\right] .
\end{aligned}
$$

\subsection{The one-loop five-gluon amplitude in the next-to-multi-Regge kinematics}

In order to extend eq. (2.2) to loop level and to NLL accuracy, we note that the fivegluon amplitude in the NMRK region of eq. (2.1) retains the crossing symmetry under $p_{b} \leftrightarrow p_{b^{\prime}}$. Using eq. (C.3), that is equivalent to a crossing symmetry of the colour-ordered amplitudes of eq. (2.2) under either $s_{2 b} \leftrightarrow s_{2 b^{\prime}}$ or $s_{1 b} \leftrightarrow s_{1 b^{\prime}}$. Then, just like for the fourgluon amplitude (1.4), but in this case for each colour-ordered amplitude, we may consider 
states whose kinematic and colour coefficients have a definite signature under the suitable crossing, extend the Reggeisation ansatz (1.10) to NMRK, and write

$$
\left.\mathcal{M}_{g g \rightarrow g g g}^{(-)\left[8_{a}\right]}\right|_{\mathrm{NMRK}}=\left.\sum_{\sigma \in S_{2}} g_{S}^{3}\left(F^{d_{\sigma_{1}}} F^{d_{\sigma_{2}}}\right)_{a c}\left(F^{b^{\prime}}\right)_{c b} m_{5 g}\left(p_{a}^{\nu_{a}}, p_{\sigma_{1}}^{\nu_{\sigma_{1}}}, p_{\sigma_{2}}^{\nu_{\sigma_{2}}}, p_{b^{\prime}}^{\nu_{b^{\prime}}}, p_{b}^{\nu_{b}}\right)\right|_{\mathrm{NMRK}},
$$

where, as in eq. (2.2), the sum is over the permutations of the labels 1 and 2, and

$$
\begin{aligned}
& \left.m_{5 g}\left(p_{a}^{\nu_{a}}, p_{1}^{\nu_{1}}, p_{2}^{\nu_{2}}, p_{b^{\prime}}^{\nu_{b^{\prime}}}, p_{b}^{\nu_{b}}\right)\right|_{\mathrm{NMRK}} \\
& =\frac{1}{2} \frac{s}{t} A^{g g}\left(p_{a}^{\nu_{a}} ; p_{1}^{\nu_{1}}, p_{2}^{\nu_{2}}\right)\left[\left(\frac{s_{2 b^{\prime}}}{\tau}\right)^{\alpha(t)}+\left(\frac{-s_{2 b^{\prime}}}{\tau}\right)^{\alpha(t)}\right] C^{g}\left(p_{b}^{\nu_{b}} ; p_{b^{\prime}}^{\nu_{b^{\prime}}}\right),
\end{aligned}
$$

where $\alpha(t)$ is given by eq. (1.11), $C^{g}\left(p_{b}^{\nu_{b}} ; p_{b^{\prime}}^{\nu_{b^{\prime}}}\right)$ by eq. (1.12), and

$$
A^{g g}\left(p_{a}^{\nu_{a}} ; p_{1}^{\nu_{1}}, p_{2}^{\nu_{2}}\right)=A^{g g(0)}\left(p_{a}^{\nu_{a}} ; p_{1}^{\nu_{1}}, p_{2}^{\nu_{2}}\right)\left(1+\frac{\alpha_{S}}{4 \pi} A_{\tilde{\nu}}^{g g(1)}\left(p_{1}, p_{2}, \tau\right)+\mathcal{O}\left(\alpha_{S}^{2}\right)\right),
$$

with $\tilde{\nu}$ in eq. (2.6). Just like in eq. (1.13), one can write the colour-ordered amplitude (2.14) as a double expansion in the strong coupling $\alpha_{S}$ and in $\log \left(s_{2 b^{\prime}} / \tau\right)$,

$$
\left.m_{5 g}\right|_{\mathrm{NMRK}}=m_{5 g}^{(0)}\left(1+\sum_{\ell=1}^{\infty}\left(\frac{\alpha_{S}}{4 \pi}\right)^{\ell} m_{5 g}^{(\ell)}\right),
$$

with $m_{5 g}^{(0)}$ as in eq. (2.3) where a NMRK subscript is understood, and where

$$
m_{5 g}^{(\ell)}=\sum_{i=0}^{\ell} \log ^{i}\left(\frac{s_{2 b^{\prime}}}{\tau}\right) m_{5 g}^{(\ell, i)},
$$

where the $m_{5 g}^{(\ell, \ell)}$ coefficients have LL accuracy, the $m_{5 g}^{(\ell, \ell-1)}$ coefficients have NLL accuracy, etc.

If at NLL accuracy only the antisymmetric octet $\boldsymbol{8}_{a}$ is exchanged in the $t$ channel, and if within $\boldsymbol{8}_{a}$ three Reggeised-gluon exchanges are missing, we expect that, just like for eq. (1.10), the real part of the five-gluon amplitude in the NMRK region of eq. (2.1) be entirely given by eq. (2.13),

$$
\operatorname{Re}\left[\mathcal{M}_{g g \rightarrow g g g}\right]_{\mathrm{NMRK}}^{\mathrm{NLL}}=\operatorname{Re}\left[\mathcal{M}_{g g \rightarrow g g g}^{(-)\left[8_{a}\right]}\right]_{\mathrm{NMRK}},
$$

where the left-hand side of eq. (2.18), once expanded at one loop, yields

$$
\operatorname{Re}\left[\mathcal{M}_{g g \rightarrow g g g}^{(1)}\right]_{\mathrm{NMRK}}^{\mathrm{NLL}}=g_{S}^{3} \frac{\alpha_{S}}{4 \pi} \sum_{\sigma \in S_{2}}\left(F^{d_{\sigma_{1}}} F^{d_{\sigma_{2}}} F^{b^{\prime}}\right)_{a b} \operatorname{Re}\left[m_{5: 1}\right]_{\mathrm{NMRK}},
$$

where the colour-ordered amplitude $m_{5: 1}$ will be provided in appendix $\mathrm{E}$ and appendix $\mathrm{F}$, where the dependence on the momenta to be permuted is understood, and where, using eqs. (2.16) and (2.18), we write

$$
\operatorname{Re}\left[m_{5: 1}\right]_{\mathrm{NMRK}}=m_{5 g}^{(0)} \operatorname{Re}\left[m_{5 g}^{(1)}\right]_{\mathrm{NMRK}},
$$



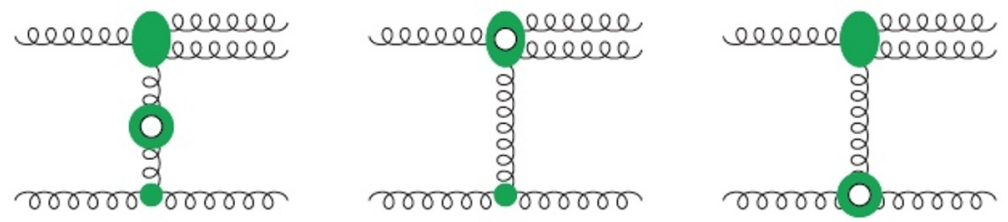

Figure 6. Factorisation of the one-loop five-gluon amplitude in NMRK. The three diagrams represent the three terms in eq. (2.20). The pierced blobs represent $(a)$ the one-loop Regge trajectory and the one-loop corrections to the impact factor for the emission of (b) two gluons and $(c)$ one gluon.

with

$$
\operatorname{Re}\left[m_{5 g}^{(1)}\right]_{\mathrm{NMRK}}=\alpha^{(1)}(t) \log \left(\frac{s_{2 b^{\prime}}}{\tau}\right)+\operatorname{Re}\left[A^{g g(1)}\left(p_{1}, p_{2}, \tau\right)\right]+\operatorname{Re}\left[C^{g(1)}(t, \tau)\right],
$$

which is displayed diagrammatically in figure 6 . The one-loop impact factors $C^{p(1)}$, with $p=g, q$, are process and infrared-scheme dependent. They were computed in conventional dimensional regularisation (CDR)/'t-Hooft-Veltman (HV) schemes in refs. [22, 28-31], and in the dimensional reduction (DR)/ four dimensional helicity (FDH) schemes in refs. [30, 31]. Through $\mathcal{O}\left(\varepsilon^{0}\right)$, the real part of the $\overline{\mathrm{MS}}$-renormalised one-loop gluon impact factor can be written as $[30,67]$

$$
\begin{aligned}
\operatorname{Re}\left[C_{-+}^{g(1)}(t, \tau)\right]=\kappa_{\Gamma}\left\{\left(\frac{\mu^{2}}{-t}\right)^{\varepsilon}\right. & {\left[-\frac{\gamma_{K}^{(1)}}{\epsilon^{2}} N_{c}+\frac{4 \gamma_{g}^{(1)}}{\epsilon}+\frac{\beta_{0}}{2 \varepsilon}+\frac{N_{c}}{\varepsilon} \log \left(\frac{\tau}{-t}\right)\right.} \\
& \left.\left.-\left(\frac{32}{9}+\frac{\delta_{R}}{6}-\frac{\pi^{2}}{2}\right) N_{c}+\frac{5}{9} N_{f}\right]-\frac{\beta_{0}}{2 \varepsilon}\right\} \\
=\kappa_{\Gamma}\left[\left(\frac{\mu^{2}}{-t}\right)^{\epsilon}\right. & {\left[\left(-\frac{2}{\epsilon^{2}}-\frac{11}{6 \varepsilon}+\frac{1}{\varepsilon} \log \left(\frac{\tau}{-t}\right)-\frac{32}{9}-\frac{\delta_{R}}{6}+\frac{\pi^{2}}{2}\right) N_{c}\right.} \\
+ & \left.\left.\left(\frac{1}{3 \varepsilon}+\frac{5}{9}\right) N_{f}\right]-\frac{\beta_{0}}{2 \epsilon}\right],
\end{aligned}
$$

with $N_{f}$ the number of light quark flavours, and where we have used the regularisation parameter, $\delta_{R}=1$ in CDR/HV schemes, $\delta_{R}=0$ in the DR/FDH schemes [68]. In eq. (2.22), the last term is the $\overline{\mathrm{MS}}$ ultraviolet counterterm, and the infrared $\varepsilon$ poles are accounted for by the cusp anomalous dimension and by the gluon collinear anomalous dimension [58], with $\gamma_{K}^{(1)}$ the one-loop coefficient of the cusp anomalous dimension (D.2), $\gamma_{g}^{(1)}$ the one-loop coefficient of the gluon collinear anomalous dimension (D.4), and $\beta_{0}$ the one-loop coefficient of the beta function (D.5).

In order to compute $A^{g g(1)}$, we use the colour decomposition of the one-loop five-gluon amplitude [69],

$$
\begin{aligned}
\mathcal{M}_{5 g}^{(1)}=g_{S}^{5} \sum_{\sigma \in S_{4} / \mathcal{R}}[ & \operatorname{Tr}\left(F^{d_{\sigma_{1}}} \ldots F^{d_{\sigma_{5}}}\right) m_{5: 1}^{[1]}\left(\sigma_{1}, \ldots, \sigma_{5}\right) \\
& \left.+2 N_{f} \operatorname{Tr}\left(T^{d_{\sigma_{1}}} \ldots T^{d_{\sigma_{5}}}\right) m_{5: 1}^{[1 / 2]}\left(\sigma_{1}, \ldots, \sigma_{5}\right)\right],
\end{aligned}
$$

where $S_{4} \equiv S_{5} / \mathbb{Z}_{5}$ is the group of the non-cyclic permutations, and $\mathcal{R}$ is the reflection: $\mathcal{R}(1, \ldots, 5)=(5, \ldots, 1)$. The superscript $[j]$ denotes the spin of the particle circulating in 
the loop. Eq. (2.23) contains 12 independent colour-ordered amplitudes of type $m_{5: 1}^{[1]}$ and 12 of type $m_{5: 1}^{[1 / 2]}$. They have been computed in ref. [65], and are recorded in appendix E in the notation of ref. [43].

We evaluate the colour-ordered amplitudes $m_{5: 1}^{[1]}$ and $m_{5: 1}^{[1 / 2]}$, appendix E, in the NMRK region of eq. (2.1) and appendix C. The leading colour-ordered amplitudes are found to be the same as at tree level (2.2), such that at one loop we can write the five-gluon colourordered amplitude as in eq. (2.19), where $m_{5: 1}$ is given by eqs. (E.8) and (F.2). Then the one-loop five-gluon amplitude is factorised as in eqs. (2.13)-(2.20).

\subsection{One-loop impact factor for the emission of two gluons}

Using eqs. (1.7), (2.20) and (2.22), we obtain from eq. (F.2) the real part of the $\overline{\mathrm{MS}}$ renormalised one-loop impact factor for the emission of two gluons,

$$
\begin{aligned}
& \frac{1}{\kappa_{\Gamma}} \operatorname{Re}\left[A_{+}^{g g(1)}\left(p_{1}, p_{2}, \tau\right)\right] \\
& =N_{c}\left\{-\frac{1}{\epsilon^{2}}\left[2\left(\frac{\mu^{2}}{\left|p_{1 \perp}\right|^{2}}\right)^{\epsilon}+\left(\frac{\mu^{2}}{\left|p_{2 \perp}\right|^{2}}\right)^{\epsilon}\right]\right. \\
& +\frac{1}{\epsilon}\left[2\left(\frac{\mu^{2}}{\left|p_{1 \perp}\right|^{2}}\right)^{\epsilon} \log \left(\frac{s_{12}}{\left|p_{1 \perp}\right|\left|p_{2 \perp}\right|}\right)+\left(\frac{\mu^{2}}{-t}\right)^{\epsilon} \log \left(\frac{\tau}{\left|p_{2 \perp}\right|^{2}}\right)\right] \\
& -\frac{1}{\epsilon}\left(\frac{\mu^{2}}{-t} \frac{\left|p_{2 \perp}\right|^{2}}{\left|p_{1 \perp}\right|^{2}}\left(\frac{p_{2}^{+}}{p_{1}^{+}} \frac{s_{12}}{\left|p_{2 \perp}\right|^{2}}\right)^{\frac{1}{2}}\right)^{\epsilon} \log \left(\frac{x_{1} x_{2} s_{12}}{\left|p_{2 \perp}\right|^{2}}\right) \\
& \left.-\frac{1}{2} \log ^{2}\left(\frac{\left|p_{1 \perp}\right|^{2}}{-t}\right)-\frac{1}{2} \log \left(x_{1}\right) \log \left(x_{1} x_{2}\left(\frac{-t}{s_{12}}\right)^{3} \frac{-t}{\left|p_{2 \perp}\right|^{2}}\right)-\frac{32}{9}-\frac{\delta_{R}}{6}+\frac{5}{6} \pi^{2}\right\} \\
& -\frac{\beta_{0}}{2 \epsilon}\left(\frac{x_{1} \mu^{2}}{\left|p_{1 \perp}\right|^{2}}\right)^{\epsilon}+\frac{5}{9} N_{f}+\frac{\beta_{0}}{2}\left(\frac{\left|p_{1 \perp}\right|^{2}}{x_{1}}-t+2 q_{\perp}^{*} p_{1 \perp}\right) \frac{L_{0}\left(\frac{\left|p_{1 \perp}\right|^{2}}{-x_{1} t}\right)}{t} \\
& +\frac{N_{C}-N_{f}}{3}\left\{\frac{p_{2}^{+}}{p_{1}^{+}}\left[\left|p_{1 \perp}\right|^{2} q_{\perp}^{* 2} p_{2 \perp}^{2}-2\left|p_{1 \perp}\right|^{2}\left|p_{2 \perp}\right|^{2} p_{1 \perp} q_{\perp}^{*}+\frac{p_{2}^{+}}{p_{1}^{+}}\left|p_{1 \perp}\right|^{4} p_{2 \perp} q_{\perp}^{*}\right]\right. \\
& \left.-\left|p_{2 \perp}\right|^{2}\left[2\left|p_{1 \perp}\right|^{2}(-t)+\left(2\left|p_{2 \perp}\right|^{2}-\left|p_{1 \perp}\right|^{2}+t\right) p_{1 \perp} q_{\perp}^{*}\right]\right\} \frac{L_{2}\left(\frac{\left|p_{1 \perp}\right|^{2}}{-x_{1} t}\right)}{t^{3}} \\
& +\frac{N_{C}-N_{f}}{6}\left[\frac{x_{2} p_{2 \perp} q_{\perp}^{*}}{-t}+\frac{2 x_{1} x_{2} q_{\perp}^{*} p_{1 \perp}^{2}}{-t\left|p_{1 \perp}\right|^{2}}-\frac{x_{1}\left|p_{2 \perp}\right|^{2} p_{1 \perp} q_{\perp}^{*}}{-t\left|p_{1 \perp}\right|^{2}}\right]-\frac{\beta_{0}}{\epsilon}+O(\epsilon),
\end{aligned}
$$

with $q_{\perp}=-\left(p_{1 \perp}+p_{2 \perp}\right)$, where the last term is the $\overline{\mathrm{MS}}$ ultraviolet counterterm, and where it is understood that in order to obtain the real part we must use eq. (F.3). The impact factor $A^{g g(1)}\left(p_{a}^{\nu_{a}} ; p_{2}^{\nu_{2}}, p_{1}^{\nu_{1}}\right)$ for the second colour ordering of eq. (2.13) is obtained by exchanging the labels 1 and 2 in eq. (2.24).

In MRK, appendix $\mathrm{B}$, with $p_{1}^{+} \gg p_{2}^{+}$, the impact factor $A^{g g}\left(p_{a}^{\nu_{a}} ; p_{2}^{\nu_{2}}, p_{1}^{\nu_{1}}\right)$ is power suppressed in $p_{2}^{+} / p_{1}^{+}$, then we just need to consider the first colour ordering in eq. (2.13), 
for which the one-loop impact factor (2.24) factorises as

$$
\lim _{p_{1}^{+} \gg p_{2}^{+}} \operatorname{Re}\left[A^{g g(1)}\left(p_{1}, p_{2}, \tau\right)\right]=\alpha^{(1)}\left(t_{1}\right) \log \left(\frac{s_{12}}{\tau}\right)+\operatorname{Re}\left[C^{g(1)}\left(t_{1}, \tau\right)\right]+\operatorname{Re}\left[V^{g(1)}\left(q_{1}, p_{2}, \tau\right)\right],
$$

with $q_{1}=-\left(p_{a}+p_{1}\right), t_{1}=q_{1}^{2} \simeq-q_{1 \perp} q_{1 \perp}^{*}$ and $q=q_{2}=q_{1}-p_{2}$, and where the one-loop corrections to the $\overline{\mathrm{MS}}$-renormalised central-emission vertex (2.11) are given by

$$
V^{g}\left(q_{1}, p_{2}\right)=V^{g(0)}\left(q_{1}, p_{2}\right)\left(1+\frac{\alpha_{S}}{4 \pi} V^{g(1)}\left(q_{1}, p_{2}, \tau\right)+\mathcal{O}\left(\alpha_{S}^{2}\right)\right),
$$

with [43]

$$
\begin{aligned}
\frac{1}{\kappa_{\Gamma}} \operatorname{Re}[ & \left.V^{g(1)}\left(q_{1}, p_{2}, \tau\right)\right] \\
= & N_{c}\left\{-\frac{1}{\epsilon^{2}}\left(\frac{\mu^{2}}{\left|p_{2 \perp}\right|^{2}}\right)^{\epsilon}+\frac{1}{\epsilon}\left[\left(\frac{\mu^{2}}{-t_{1}}\right)^{\epsilon}+\left(\frac{\mu^{2}}{-t_{2}}\right)^{\epsilon}\right] \log \left(\frac{\tau}{\left|p_{2 \perp}\right|^{2}}\right)-\frac{1}{2} \log ^{2}\left(\frac{t_{1}}{t_{2}}\right)+\frac{\pi^{2}}{3}\right\} \\
& -\frac{\beta_{0}}{2}\left(t_{1}+t_{2}+2 q_{1 \perp} q_{2 \perp}^{*}\right) \frac{L_{0}\left(t_{1} / t_{2}\right)}{t_{2}} \\
& +\frac{N_{c}-N_{f}}{3}\left|p_{2 \perp}\right|^{2}\left[-\left[2 t_{1} t_{2}+\left(t_{1}+t_{2}+2\left|p_{2 \perp}\right|^{2}\right) q_{1 \perp} q_{2 \perp}^{*}\right] \frac{L_{2}\left(t_{1} / t_{2}\right)}{t_{2}^{3}}-\frac{q_{1 \perp} q_{2 \perp}^{*}}{2 t_{1} t_{2}}\right]-\frac{\beta_{0}}{2 \epsilon} \\
& +O(\epsilon),
\end{aligned}
$$

with $t=t_{2}=q_{2}^{2}$, where the last term is the $\overline{\mathrm{MS}}$ ultraviolet counterterm, and where it is understood that in order to obtain the real part we must use

$$
2 \operatorname{Re}\left(q_{1 \perp} q_{2 \perp}^{*}\right)=-\left(t_{1}+t_{2}+\left|p_{2 \perp}\right|^{2}\right) .
$$

\subsection{One-loop two-quark three-gluon amplitude in NMRK}

Regge factorisation at tree level implies that in the NMRK region of eq. (2.1), we can write the two-quark three-gluon tree amplitude for the $g_{a} q_{b} \rightarrow g_{1} g_{2} q_{b^{\prime}}$ scattering, figure 5(b), as $[40,62]$

$$
\left.\mathcal{M}_{g q \rightarrow g g q}^{(0)}\right|_{\mathrm{NMRK}}=\left.\sum_{\sigma \in S_{2}} g_{S}^{3}\left(F^{d_{\sigma_{1}}} F^{d_{\sigma_{2}}}\right)_{a c}\left(T^{c}\right)_{b^{\prime} \bar{b}} m_{2 q 3 g}^{(0)}\left(p_{a}^{\nu_{a}}, p_{\sigma_{1}}^{\nu_{\sigma_{1}}}, p_{\sigma_{2}}^{\nu_{\sigma_{2}}}, p_{b^{\prime}}^{\nu_{b^{\prime}}}, p_{b}^{\nu_{b}}\right)\right|_{\mathrm{NMRK}},
$$

where the sum is over the permutations of the labels 1 and 2, and the colour-ordered amplitude is

$$
\left.m_{2 q 3 g}^{(0)}\left(p_{a}^{\nu_{a}}, p_{1}^{\nu_{1}}, p_{2}^{\nu_{2}}, p_{b^{\prime}}^{\nu_{b^{\prime}}}, p_{b}^{\nu_{b}}\right)\right|_{\mathrm{NMRK}}=A^{g g(0)}\left(p_{a}^{\nu_{a}} ; p_{1}^{\nu_{1}}, p_{2}^{\nu_{2}}\right) \frac{s}{t} C^{q(0)}\left(p_{b}^{\nu_{b}} ; p_{b^{\prime}}^{\nu_{b^{\prime}}}\right),
$$

where $A^{g g(0)}$ is given by eq. (2.4), and the quark impact factor is ${ }^{6}$

$$
C^{q(0)}\left(p_{b}^{-} ; p_{b^{\prime}}^{+}\right)=i \sqrt{\frac{p_{b^{\prime} \perp}^{*}}{p_{b^{\prime} \perp}}},
$$

\footnotetext{
${ }^{6}$ Since helicities are for all outgoing momenta, an incoming right-handed quark is labelled in eq. (2.31) and the right-hand side of eq. (2.29) as a left-handed antiquark, so e.g. the amplitude $g_{R}\left(-p_{a}\right) q_{R}\left(-p_{b}\right) \rightarrow$ $g_{R}\left(p_{1}\right) g_{R}\left(p_{2}\right) q_{R}\left(p_{b^{\prime}}\right)$ becomes $\mathcal{M}\left(a_{g}^{-}, 1_{g}^{+}, 2_{g}^{+}, b_{q}^{\prime+}, b_{\bar{q}}^{-}\right)$.
} 
which under parity becomes [62]

$$
\left[C^{q(0)}\left(p_{b}^{-\nu} ; p_{b^{\prime}}^{\nu}\right)\right]^{*}=S C^{q(0)}\left(p_{b}^{\nu} ; p_{b^{\prime}}^{-\nu}\right) \quad \text { with } \quad S=-\operatorname{sign}\left(\bar{q}^{0} q^{0}\right)
$$

If Regge factorisation holds at NLL accuracy, then we can write the two-quark three-gluon amplitude in the NMRK region of eq. (2.1) as

$$
\left.\mathcal{M}_{g q \rightarrow g g q}^{(-)\left[8_{a}\right]}\right|_{\mathrm{NMRK}}=\left.\sum_{\sigma \in S_{2}} g_{S}^{3}\left(F^{d_{\sigma_{1}}} F^{d_{\sigma_{2}}}\right)_{a c}\left(T^{c}\right)_{b^{\prime} \bar{b}} m_{2 q 3 g}\left(p_{a}^{\nu_{a}}, p_{\sigma_{1}}^{\nu_{\sigma_{1}}}, p_{\sigma_{2}}^{\nu_{\sigma_{2}}}, p_{b^{\prime}}^{\nu_{b^{\prime}}}, p_{b}^{\nu_{b}}\right)\right|_{\mathrm{NMRK}}
$$

with the colour-ordered amplitude,

$$
\begin{aligned}
\left.m_{2 q 3 g}\left(p_{a}^{\nu_{a}}, p_{1}^{\nu_{1}}, p_{2}^{\nu_{2}}, p_{b^{\prime}}^{\nu_{b^{\prime}}}, p_{b}^{\nu_{b}}\right)\right|_{\mathrm{NMRK}} & \\
& =\frac{1}{2} \frac{s}{t} A^{g g}\left(p_{a}^{\nu_{a}} ; p_{1}^{\nu_{1}}, p_{2}^{\nu_{2}}\right)\left[\left(\frac{s_{2 b^{\prime}}}{\tau}\right)^{\alpha(t)}+\left(\frac{-s_{2 b^{\prime}}}{\tau}\right)^{\alpha(t)}\right] C^{q}\left(p_{b}^{\nu_{b}} ; p_{b^{\prime}}^{\nu_{b^{\prime}}}\right),
\end{aligned}
$$

where $A^{g g}\left(p_{a}^{\nu_{a}} ; p_{1}^{\nu_{1}}, p_{2}^{\nu_{2}}\right)$ is given by eq. (2.15), and $C^{q}\left(p_{b}^{\nu_{b}} ; p_{b^{\prime}}^{\nu_{b^{\prime}}}\right)$ is given by the quark analog of eq. (1.12). We can then write the colour-ordered amplitude (2.34) as a double expansion in the strong coupling $\alpha_{S}$ and in $\log \left(s_{2 b^{\prime}} / \tau\right)$, and repeat the steps of eq. (2.16) through eq. (2.20). Once expanded at one loop, the real part of eq. (2.34) becomes

$$
\operatorname{Re}\left[m_{2 q 3 g}^{(1)}\right]_{\mathrm{NMRK}}=\alpha^{(1)}(t) \log \left(\frac{s_{\sigma_{2} b^{\prime}}}{\tau}\right)+\operatorname{Re}\left[A^{g g(1)}\left(p_{\sigma_{1}}, p_{\sigma_{2}}, \tau\right)\right]+\operatorname{Re}\left[C^{q(1)}(t, \tau)\right],
$$

with $A^{g g(1)}$ as in eq. (2.24), and where, through $\mathcal{O}\left(\varepsilon^{0}\right)$, the $\overline{\mathrm{MS}}$-renormalised one-loop quark impact factor $C^{q(1)}(t)$ can be written as $[30,67]$

$$
\begin{aligned}
& \operatorname{Re}\left[C_{-+}^{q(1)}(t, \tau)\right]=\kappa_{\Gamma}\left\{\left(\frac{\mu^{2}}{-t}\right)^{\epsilon}\right. {\left[-\frac{\gamma_{K}^{(1)}}{\epsilon^{2}} C_{F}+\frac{4}{\varepsilon} \gamma_{q}^{(1)}+\frac{\beta_{0}}{2 \varepsilon}+\frac{N_{c}}{\varepsilon} \log \left(\frac{\tau}{-t}\right)\right.} \\
&+\left.\left.\left(\frac{19}{18}-\frac{\delta_{R}}{3}+\frac{\pi^{2}}{2}\right) N_{c}-\frac{5}{9} N_{f}+\left(\frac{7}{2}+\frac{\delta_{R}}{2}\right) \frac{1}{N_{c}}\right]-\frac{\beta_{0}}{2 \varepsilon}\right\} \\
&=\kappa_{\Gamma}\left\{( \frac { \mu ^ { 2 } } { - t } ) ^ { \epsilon } \left[\left(-\frac{1}{\epsilon^{2}}+\frac{1}{3 \epsilon}+\frac{1}{\varepsilon} \log \left(\frac{\tau}{-t}\right)+\frac{19}{18}-\frac{\delta_{R}}{3}+\frac{\pi^{2}}{2}\right) N_{c}\right.\right. \\
&\left.\left.-\left(\frac{1}{3 \epsilon}+\frac{5}{9}\right) N_{f}+\left(\frac{1}{\epsilon^{2}}+\frac{3}{2 \epsilon}+\frac{7}{2}+\frac{\delta_{R}}{2}\right) \frac{1}{N_{c}}\right]-\frac{\beta_{0}}{2 \epsilon}\right\},
\end{aligned}
$$

with $\gamma_{q}^{(1)}$ the one-loop coefficient of the quark collinear anomalous dimension (D.4).

In order to verify that the one-loop two-quark three-gluon amplitude in NMRK takes the factorised form given by eq. (2.35), we use the colour decomposition of the one-loop 
two-quark three-gluon amplitude [69],

$$
\begin{aligned}
M_{2 q 3 g}^{1-\mathrm{loop}}\left(1_{\bar{q}}, 2_{q}, 3,4,5\right) & \\
=g_{S}^{5}[ & \sum_{p=2}^{5} \sum_{\sigma \in S_{3}}\left(T^{c_{2}} T^{d_{\sigma_{3}}} \ldots T^{d_{\sigma_{p}}} T^{c_{1}}\right)_{i_{2}}^{\bar{i}_{1}}\left(F^{d_{\sigma_{p+1}}} \ldots F^{d_{\sigma_{5}}}\right)_{c_{1} c_{2}} \\
& \times A_{5}^{R,[1]}\left(1_{\bar{q}}, \sigma_{p+1}, \ldots, \sigma_{5}, 2_{q}, \sigma_{3}, \ldots, \sigma_{p}\right) \\
& \left.+\frac{N_{f}}{N_{c}} \sum_{j=1}^{4} \sum_{\sigma \in S_{3} / S_{5 ; j}} G r_{5 ; j}^{\bar{q} q}\left(\sigma_{3}, \sigma_{4}, \sigma_{5}\right) A_{5 ; j}^{[1 / 2]}\left(1_{\bar{q}}, 2_{q}, \sigma_{3}, \sigma_{4}, \sigma_{5}\right)\right],
\end{aligned}
$$

where the colour structures $G r_{5 ; j}^{\bar{q} q}$ are defined by

$$
\begin{aligned}
& G r_{5 ; 1}^{\bar{q} q}(3,4,5)=N_{c}\left(T^{d_{3}} T^{d_{4}} T^{d_{5}}\right)_{i_{2}}^{\bar{i}_{1}}, \\
& G r_{5 ; 2}^{\bar{q} q}(3 ; 4,5)=0, \\
& G r_{5 ; 3}^{\bar{q} q}(3,4 ; 5)=\operatorname{Tr}\left(T^{d_{3}} T^{d_{4}}\right)\left(T^{d_{5}}\right)_{i_{2}}^{\bar{i}_{1}}, \\
& G r_{5 ; 4}^{\bar{q} q}(3,4 ; 5)=\operatorname{Tr}\left(T^{d_{3}} T^{d_{4}} T^{d_{5}}\right) \delta_{i_{2}}^{\bar{i}_{1}},
\end{aligned}
$$

and $S_{5 ; j}=\mathbb{Z}_{j-1}$ is the subgroup of $S_{3}$ that leaves $G r_{5 ; j}^{\bar{q} q}$ invariant, and

$$
\begin{aligned}
& A_{5 ; 1}^{[1 / 2]}\left(1_{\bar{q}}, 2_{q}, 3,4,5\right)=A_{5}^{L,[1 / 2]}\left(1_{\bar{q}}, 2_{q}, 3,4,5\right), \\
& A_{5 ; 3}^{[1 / 2]}\left(1_{\bar{q}}, 2_{q} ; 3,4 ; 5\right)=-\sum_{\sigma \in\{4,3\} \amalg\{1,2,5\}} A_{5 ; 1}^{[1 / 2]}\left(\sigma\left(1_{\bar{q}}, 2_{q}, 3,4,5\right)\right), \\
& A_{5 ; 4}^{[1 / 2]}\left(1_{\bar{q}}, 2_{q} ; 3,4 ; 5\right)=\sum_{\sigma \in\{5,4,3\} \amalg\{1,2\}} A_{5 ; 1}^{[1 / 2]}\left(\sigma\left(1_{\bar{q}}, 2_{q}, 3,4,5\right)\right),
\end{aligned}
$$

where the shuffle product $\{\alpha\} \amalg\{\beta\}$ yields the permutations which preserve the orderings of the elements in $\{\alpha\}$ and in $\{\beta\}$ while allowing for all possible orderings of the elements of $\{\alpha\}$ with respect to $\{\beta\}$. In eq. (2.39), we did not list the $j=2$ case, since the corresponding colour structure is missing in eq. (2.38). Further, we spell out the first sum of eq. (2.37),

$$
\begin{aligned}
& \sum_{p=2}^{5} \sum_{\sigma \in S_{3}}\left(T^{c_{2}} T^{d_{\sigma_{3}}} \ldots T^{d_{\sigma_{p}}} T^{c_{1}}\right)_{i_{2}}^{\bar{i}_{1}}\left(F^{d_{\sigma_{p+1}}} \ldots F^{d_{\sigma_{5}}}\right)_{c_{1} c_{2}} \\
& \times A_{5}^{R,[1]}\left(1_{\bar{q}}, \sigma_{p+1}, \ldots, \sigma_{5}, 2_{q}, \sigma_{3}, \ldots, \sigma_{p}\right) \\
& =\sum_{\sigma \in S_{3}}\left[\left(T^{c_{2}} T^{c_{1}}\right)_{i_{2}}^{\bar{i}_{1}}\left(F^{d_{\sigma_{3}}} F^{d_{\sigma_{4}}} F^{d_{\sigma_{5}}}\right)_{c_{1} c_{2}} A_{5}^{R,[1]}\left(1_{\bar{q}}, \sigma_{3}, \sigma_{4}, \sigma_{5}, 2_{q}\right)\right. \\
& +\left(T^{c_{2}} T^{d_{\sigma_{3}}} T^{c_{1}}\right)_{i_{2}}^{\bar{i}_{1}}\left(F^{d_{\sigma_{4}}} F^{d_{\sigma_{5}}}\right)_{c_{1} c_{2}} A_{5}^{R,[1]}\left(1_{\bar{q}}, \sigma_{4}, \sigma_{5}, 2_{q}, \sigma_{3}\right) \\
& +\left(T^{c_{2}} T^{d_{\sigma_{3}}} T^{d_{\sigma_{4}}} T^{c_{1}}\right)_{i_{2}}^{\bar{i}_{1}}\left(F^{d_{\sigma_{5}}}\right)_{c_{1} c_{2}} A_{5}^{R,[1]}\left(1_{\bar{q}}, \sigma_{5}, 2_{q}, \sigma_{3}, \sigma_{4}\right) \\
& \left.+\left(T^{c_{2}} T^{d_{\sigma_{3}}} T^{d_{\sigma_{4}}} T^{d_{\sigma_{5}}} T^{c_{1}}\right)_{i_{2}}^{\bar{i}_{1}} \delta_{c_{1} c_{2}} A_{5}^{R,[1]}\left(1_{\bar{q}}, 2_{q}, \sigma_{3}, \sigma_{4}, \sigma_{5}\right)\right] .
\end{aligned}
$$


Finally, the reflection identity,

$$
A_{n}^{R,[1]}\left(1_{\bar{q}}, 3, \ldots, 2_{q}, \ldots, n-1, n\right)=(-1)^{n} A_{n}^{L,[1]}\left(1_{\bar{q}}, n, n-1, \ldots, 2_{q}, \ldots, 3\right),
$$

relates the left and right primitive amplitudes.

The primitive amplitudes $A_{5}^{L,[1]}\left(1_{\bar{q}}, 2_{q}, 3,4,5\right)$ and $A_{5}^{L,[1 / 2]}\left(1_{\bar{q}}, 2_{q}, 3,4,5\right)$, which are necessary to assemble the one-loop two-quark three-gluon amplitude (2.37), have been provided by ref. [66] in the DR/FDH schemes. We have evaluated them in the NMRK region of eq. (2.1) and appendix C. In NMRK, the leading contributions are given by the terms with $p=2, p=5$ and $j=1$ in eq. (2.37), i.e. by the first and the last term on the right-hand side of eq. (2.40) and by the first term of eqs. (2.38) and (2.39).

\subsubsection{The $p=2$ term}

The knowledge of the primitive amplitudes $A_{5}^{L,[1]}\left(1_{\bar{q}}, 2_{q}, 3,4,5\right)[66]$, and the reflection identity (2.41) allow us to compute the primitive amplitudes $A_{5}^{R,[1]}$, which, in the notation of the $g_{a} q_{b} \rightarrow g_{1} g_{2} q_{b^{\prime}}$ scattering, are $A_{5}^{R,[1]}\left(b_{\bar{q}}, \sigma_{a}, \sigma_{1}, \sigma_{2}, b_{q}^{\prime}\right)$. Computing the primitive amplitudes $A_{5}^{R,[1]}$ in the NMRK of eq. (2.1) for different colour orderings, we find that they are related by

$$
\begin{aligned}
A_{5}^{R,[1]}\left(b_{\bar{q}}, a, 1,2, b_{q}^{\prime}\right) & =A_{5}^{R,[1]}\left(b_{\bar{q}}, 2,1, a, b_{q}^{\prime}\right)=-\lambda_{1} m_{2 q 3 g}^{(0)}\left(p_{a}, p_{1}, p_{2}, p_{b^{\prime}}, p_{b}\right) \\
A_{5}^{R,[1]}\left(b_{\bar{q}}, a, 2,1, b_{q}^{\prime}\right) & =A_{5}^{R,[1]}\left(b_{\bar{q}}, 1,2, a, b_{q}^{\prime}\right)=-\lambda_{2} m_{2 q 3 g}^{(0)}\left(p_{a}, p_{2}, p_{1}, p_{b^{\prime}}, p_{b}\right) \\
A_{5}^{R,[1]}\left(b_{\bar{q}}, 1, a, 2, b^{\prime}\right) & =A_{5}^{R,[1]}\left(b_{\bar{q}}, 2, a, 1, b_{q}^{\prime}\right) \\
& =\lambda_{1} m_{2 q 3 g}^{(0)}\left(p_{a}, p_{1}, p_{2}, p_{b^{\prime}}, p_{b}\right)+\lambda_{2} m_{2 q 3 g}^{(0)}\left(p_{a}, p_{2}, p_{1}, p_{b^{\prime}}, p_{b}\right)
\end{aligned}
$$

with $\lambda_{1}, \lambda_{2}$ in eqs. (G.1) and (G.2), and $m_{2 q 3 g}^{(0)}\left(p_{a}, p_{2}, p_{1}, p_{b^{\prime}}, p_{b}\right)$ the tree colour-ordered amplitude (2.30), where a NMRK subscript is understood. Using eq. (2.42), the colour coefficient of the $p=2$ term of eq. (2.37), i.e. of the first term on the right-hand side of eq. (2.40), simplifies and factors an $N_{c}$ term out. It can be written as

$$
\begin{aligned}
\sum_{\sigma \in S_{3}}\left(T^{c_{2}} T^{c_{1}}\right)_{b^{\prime}}^{\bar{b}}\left(F^{d_{\sigma_{a}}} F^{d_{\sigma_{1}}} F^{d_{\sigma_{2}}}\right)_{c_{1} c_{2}} A_{5}^{R,[1]}\left(b_{\bar{q}}, \sigma_{a}, \sigma_{1}, \sigma_{2}, b_{q}^{\prime}\right) \\
\stackrel{\mathrm{NMRKK}}{\Longrightarrow} N_{c} \sum_{\sigma \in S_{2}}\left(F^{d_{\sigma_{1}}} F^{d_{\sigma_{2}}}\right)_{a c}\left(T^{c}\right)_{b^{\prime}}^{\bar{b}} \lambda_{\sigma_{1}} m_{2 q 3 g}^{(0)}\left(p_{a}, p_{\sigma_{1}}, p_{\sigma_{2}}, p_{b^{\prime}}, p_{b}\right) .
\end{aligned}
$$

Thus, the $p=2$ term provides the leading $N_{c}$ terms of the one-loop two-quark three-gluon amplitude in NMRK.

\subsubsection{The $p=5$ term}

For the $p=5$ term, one can use the relation [66],

$$
\begin{aligned}
& A_{5}^{R,[1]}\left(b_{\bar{q}}, b^{\prime}, \sigma_{a}, \sigma_{1}, \sigma_{2}\right) \\
& \quad=A_{5}^{\mathrm{SUSY}}\left(b_{\bar{q}}, b^{\prime}{ }_{q}, \sigma_{a}, \sigma_{1}, \sigma_{2}\right)-A_{5}^{L,[1]}\left(b_{\bar{q}}, b_{q}^{\prime}{ }_{q}, \sigma_{a}, \sigma_{1}, \sigma_{2}\right)-A_{5}^{L,[1 / 2]}\left(b_{\bar{q}}, b_{q}^{\prime}, \sigma_{a}, \sigma_{1}, \sigma_{2}\right),
\end{aligned}
$$


where the primitive amplitudes on the right-hand side are all provided in ref. [66]. Computing the primitive amplitudes $A_{5}^{R,[1]}$ in the NMRK of eq. (2.1) for different colour orderings, we find that they are related by

$$
\begin{aligned}
A_{5}^{R,[1]}\left(b_{\bar{q}}, b_{q}^{\prime}, a, 1,2\right) & =A_{5}^{R,[1]}\left(b_{\bar{q}}, b_{q}^{\prime}, 2,1, a\right)=\Phi m_{2 q 3 g}^{(0)}\left(p_{a}, p_{1}, p_{2}, p_{b}, p_{b^{\prime}}\right), \\
A_{5}^{R,[1]}\left(b_{\bar{q}}, b_{q}^{\prime}, a, 2,1\right) & =A_{5}^{R,[1]}\left(b_{\bar{q}}, b_{q}^{\prime}, 1,2, a\right)=\Phi m_{2 q 3 g}^{(0)}\left(p_{a}, p_{2}, p_{1}, p_{b}, p_{b^{\prime}}\right), \\
A_{5}^{R,[1]}\left(b_{\bar{q}}, b_{q}^{\prime}, 1, a, 2\right) & =A_{5}^{R,[1]}\left(b_{\bar{q}}, b_{q}^{\prime}, 2, a, 1\right) \\
& =-\Phi\left[m_{2 q 3 g}^{(0)}\left(p_{a}, p_{1}, p_{2}, p_{b}, p_{b^{\prime}}\right)+m_{2 q 3 g}^{(0)}\left(p_{a}, p_{2}, p_{1}, p_{b}, p_{b^{\prime}}\right)\right],
\end{aligned}
$$

where $m_{2 q 3 g}^{(0)}\left(p_{a}, p_{1}, p_{2}, p_{b}, p_{b^{\prime}}\right)=-m_{2 q 3 g}^{(0)}\left(p_{a}, p_{1}, p_{2}, p_{b^{\prime}}, p_{b}\right)$, and with

$$
\Phi=\kappa_{\Gamma}\left(\frac{\mu^{2}}{-t}\right)^{\epsilon}\left(\frac{1}{\epsilon^{2}}+\frac{3}{2 \epsilon}+\frac{7}{2}\right) .
$$

Using eq. (2.45), the colour coefficient of the $p=5$ term of eq. (2.37), i.e. of the fourth term on the right-hand side of eq. (2.40), simplifies and factors a $1 / N_{c}$ term out. It can be written as

$$
\begin{aligned}
\sum_{\sigma \in S_{3}} \delta_{c_{1} c_{2}}\left(T^{c_{2}} T^{d_{\sigma_{a}}} T^{d_{\sigma_{1}}} T^{d_{\sigma_{2}}} T^{c_{1}}\right)_{b^{\prime}}^{\bar{b}} A_{5}^{R,[1]}\left(b_{\bar{q}}, b_{q}^{\prime}, \sigma_{a}, \sigma_{1}, \sigma_{2}\right) \\
\quad \stackrel{\mathrm{NMRK}}{\Longrightarrow} \frac{1}{N_{c}} \Phi \sum_{\sigma \in S_{2}}\left(F^{d_{\sigma_{1}}} F^{d_{\sigma_{2}}}\right)_{a c}\left(T^{c}\right)_{b^{\prime}}^{\bar{b}} \lambda_{\sigma_{1}} m_{2 q 3 g}^{(0)}\left(p_{a}, p_{\sigma_{1}}, p_{\sigma_{2}}, p_{b}, p_{b^{\prime}}\right) .
\end{aligned}
$$

Thus, the $p=5$ term provides the $1 / N_{c}$ terms of the one-loop two-quark three-gluon amplitude in NMRK.

\subsubsection{The $j=1$ term}

For the $j=1$ term, we use the first term of eqs. (2.38) and (2.39). Computing the primitive amplitudes $A_{5 ; 1}^{[1 / 2]}$ in the NMRK of eq. (2.1) for different colour orderings, we find that they are related by,

$$
\begin{aligned}
A_{5 ; 1}^{[1 / 2]}\left(b_{\bar{q}}, b_{q}^{\prime}, a, 1,2\right) & =A_{5 ; 1}^{[1 / 2]}\left(b_{\bar{q}}, b_{q}^{\prime}, 2,1, a\right)=\chi_{1} m_{2 q 3 g}^{(0)}\left(p_{a}, p_{1}, p_{2}, p_{b}, p_{b^{\prime}}\right) \\
A_{5 ; 1}^{[1 / 2]}\left(b_{\bar{q}}, b_{q}^{\prime}, a, 2,1\right) & =A_{5 ; 1}^{[1 / 2]}\left(b_{\bar{q}}, b_{q}^{\prime}, 1,2, a\right)=\chi_{2} m_{2 q 3 g}^{(0)}\left(p_{a}, p_{2}, p_{1}, p_{b}, p_{b^{\prime}}\right), \\
A_{5 ; 1}^{[1 / 2]}\left(b_{\bar{q}}, b_{q}^{\prime}, 1, a, 2\right) & =A_{5 ; 1}^{[1 / 2]}\left(b_{\bar{q}}, b_{q}^{\prime}, 2, a, 1\right) \\
& =-\chi_{1} m_{2 q 3 g}^{(0)}\left(p_{a}, p_{1}, p_{2}, p_{b}, p_{b^{\prime}}\right)-\chi_{2} m_{2 q 3 g}^{(0)}\left(p_{a}, p_{2}, p_{1}, p_{b}, p_{b^{\prime}}\right),
\end{aligned}
$$

with $\chi_{1}, \chi_{2}$ in eqs. (G.3) and (G.4). Using eq. (2.48), the colour coefficient of the $j=1$ term of eq. (2.37) simplifies and can be written as

$$
\begin{aligned}
N_{f} \sum_{\sigma \in S_{3}}\left(T^{d_{\sigma_{a}}} T^{d_{\sigma_{1}}} T^{d_{\sigma_{2}}}\right)_{b^{\prime}}^{\bar{b}} A_{5 ; 1}^{[1 / 2]}\left(b_{\bar{q}}, b_{q}^{\prime}, \sigma_{a}, \sigma_{1}, \sigma_{2}\right) \\
\stackrel{\stackrel{\mathrm{NMRK}}{\longrightarrow}}{\Longrightarrow} N_{f} \sum_{\sigma \in S_{2}}\left(F^{d_{\sigma_{1}}} F^{d_{\sigma_{2}}}\right)_{a c}\left(T^{c}\right)_{b^{\prime}}^{\bar{b}} \chi_{\sigma_{1}} m_{2 q 3 g}^{(0)}\left(p_{a}, p_{\sigma_{1}}, p_{\sigma_{2}}, p_{b}, p_{b^{\prime}}\right) .
\end{aligned}
$$

Thus, the $j=1$ term provides the $N_{f}$ terms of the one-loop two-quark three-gluon amplitude in NMRK. 


\subsubsection{Regge factorisation}

Combining eqs. (2.43) and (2.47) and (2.49), we obtain the one-loop two-quark three-gluon amplitude in the NMRK region of eq. (2.1),

$$
\begin{aligned}
& \operatorname{Re}\left[\mathcal{M}_{g q \rightarrow g g q}^{(1)}\right]_{\mathrm{NMRK}} \\
& \quad=\sum_{\sigma \in S_{2}}\left(F^{d_{\sigma_{1}}} F^{d_{\sigma_{2}}}\right)_{a c}\left(T^{c}\right)_{b^{\prime}}^{\bar{b}}\left(N_{c} \lambda_{\sigma_{1}}+N_{f} \chi_{\sigma_{1}}+\frac{\Phi}{N_{c}}\right) m_{2 q 3 g}^{(0)}\left(p_{a}, p_{\sigma_{1}}, p_{\sigma_{2}}, p_{b^{\prime}}, p_{b}\right) .
\end{aligned}
$$

Then we use eq. (2.35), with the one-loop Regge trajectory (1.7) and the one-loop quark impact factor (2.36), which in particular subtracts out all the $1 / N_{c}$ terms, and we obtain the one-loop impact factor for the emission of two gluons $A^{g g(1)}$, which is in agreement with eq. (2.24), thus verifying that Regge factorisation holds at NLL accuracy in NMRK, i.e. that at NLL accuracy only the antisymmetric octet $\mathbf{8}_{a}$ is exchanged in the $t$ channel, and within $\boldsymbol{8}_{a}$ three Reggeised-gluon exchanges are missing.

\subsection{Helicity-violating contributions}

The conservation of helicity along the $s$-channel direction in Minkowski space, that we mentioned in the Introduction, is an approximate feature of the Regge limit of tree fourgluon amplitudes, since the helicity-flip impact factor $C^{g(0)}\left(p^{+} ; p^{+}\right)$exists, but is power suppressed in $t / s$. At one loop, the four-gluon amplitude $m_{4}^{(1)}(-,+,+,+)$ is not vanishing, so the helicity-flip impact factor $C^{g(1)}\left(p^{+} ; p^{+}\right)$is not power suppressed in $t / s[22,28,30]$. In fact, we can write

$$
C^{g}\left(p_{j}^{+} ; p_{j^{\prime}}^{+}\right)=C^{g(0)}\left(p_{j}^{-} ; p_{j^{\prime}}^{+}\right)\left(\frac{\alpha_{S}}{4 \pi} C_{++}^{g(1)}\left(q_{\perp}\right)+\mathcal{O}\left(\alpha_{S}^{2}\right)\right),
$$

with $j=a, b$ and $q_{\perp}=p_{b^{\prime} \perp}=-p_{a^{\prime} \perp}$, and

$$
C_{++}^{g(1)}\left(q_{\perp}\right)=-\frac{N_{c}-N_{f}}{3} \frac{q_{\perp}^{*}}{q_{\perp}} .
$$

Because the tree four-gluon amplitude $m_{4}^{(0)}(-,+,+,+)$ vanishes, the one loop four-gluon amplitude $m_{4}^{(1)}(-,+,+,+)$ contributes to a squared amplitude, and so to a cross section, only when it is squared by itself. Thus, although the helicity-flip impact factor $C^{g}\left(p^{+} ; p^{+}\right)$ contributes to the four-gluon amplitude at NLL accuracy, it occurs in a squared amplitude only at NNLL accuracy. As such, it will contribute to the computation of the jet impact factor at NNLO in $\alpha_{S}$, mentioned at the end of section 1.3.

Likewise, although the tree five-gluon amplitude $m_{5}^{(0)}(-,+,+,+,+)$ vanishes, the one loop amplitude $m_{5}^{(1)}(-,+,+,+,+)$ does not. In general kinematics, its expression is given in ref. [65]. In the NMRK region of eq. (2.1), it becomes

$$
\begin{aligned}
& M_{5: 1}^{[1]}\left(b^{-}, a^{+}, 1^{+}, 2^{+}, b^{\prime+}\right) \\
& \quad=\frac{g_{S}^{2}}{48 \pi^{2}}\left(N_{c}-N_{f}\right) M_{5}^{(0)}\left(b^{-}, a^{-}, 1^{+}, 2^{+}, b^{\prime+}\right)\left(-x_{2} \frac{p_{b^{\prime} \perp}^{*}}{p_{b^{\prime} \perp}}-x_{1} \frac{p_{1 \perp}^{*}}{p_{1 \perp}}+x_{1} x_{2} \frac{[12]^{2}}{s_{12}}\right),
\end{aligned}
$$


with $x_{1,2}$ in eq. (2.8). From eq. (2.53) we may derive the helicity-flip impact factor $A^{g g}\left(p_{a}^{+} ; p_{1}^{+}, p_{2}^{+}\right)$for the emission of two gluons,

$$
A^{g g}\left(p_{a}^{+} ; p_{1}^{+}, p_{2}^{+}\right)=A^{g g(0)}\left(p_{a}^{-} ; p_{1}^{+}, p_{2}^{+}\right)\left(\frac{\alpha_{S}}{4 \pi} \bar{A}_{+}^{g g(1)}\left(p_{1}, p_{2}\right)+\mathcal{O}\left(\alpha_{S}^{2}\right)\right),
$$

with

$$
\bar{A}_{+}^{g g(1)}\left(p_{1}, p_{2}\right)=\frac{N_{c}-N_{f}}{3}\left(-x_{2} \frac{q_{\perp}^{*}}{q_{\perp}}-x_{1} \frac{p_{1 \perp}^{*}}{p_{1 \perp}}+x_{1} x_{2} \frac{[12]^{2}}{s_{12}}\right),
$$

with $q_{\perp}=p_{b^{\prime} \perp}=-\left(p_{1 \perp}+p_{2 \perp}\right)$. Note however that although the helicity-flip impact factor $A^{g g}\left(p_{a}^{+} ; p_{1}^{+}, p_{2}^{+}\right)$contributes to the five-gluon amplitude at NLL accuracy, it contributes to the jet impact factor only at next-to-next-to-next-to-leading order $\left(\mathrm{N}^{3} \mathrm{LO}\right)$ in $\alpha_{S}$.

\section{Discussion and conclusions}

In the Regge limit of QCD amplitudes, large logarithms $\log (s / t)$ arise. For octet exchange in the $t$ channel, they are resummed by the BFKL equation at leading logarithmic and NLL accuracy. Also the other colour representations, which are exchanged in the $t$ channel of gluon-gluon scattering amplitudes, have been fully analysed at NLL accuracy [7, 49-51]. Moving toward a BFKL equation at NNLL accuracy presents several challenges, which go from taking stock of the exchange of three Reggeised gluons [49, 52-54], which violate Regge-pole factorisation [27], to computing loop-level building blocks of the BFKL kernel in multi-Regge and next-to-multi-Regge kinematics.

In this paper, we have evaluated the one-loop impact factors for the emission of two gluons, eqs. (2.24) and (2.55), by taking the one-loop five-gluon [65] and two-quark threegluon amplitudes [66] in NMRK. As a sanity check, we verified that in MRK, eq. (F.2) is reduced to the one-loop five-gluon amplitude which yields the one-loop central-emission vertex [43].

The form of eq. (2.24) depends on the Reggeisation ansatz (2.13). In eq. (2.13), we have chosen a different base of the Regge-trajectory exponent for each of the two colourordered amplitudes. Such issue is immaterial in the Regge limit (1.10) or in MRK [43], where there is only one colour-ordered amplitude. The ansatz (2.13) ensures that in the MRK limit the impact factor (2.24) has the correct factorisation properties, i.e. that it is reduced to eq. (2.25). We note that in eq. (2.13) one could avoid introducing a different base of the trajectory exponent for each colour-ordered amplitude, by taking as a base the geometric $\frac{\sqrt{s_{1 b^{\prime}} s_{2 b^{\prime}}}}{\tau}$ or the arithmetic $\frac{s_{1 b^{\prime}}+s_{2 b^{\prime}}}{2 \tau}$ means of $s_{1 b^{\prime}}$ and $s_{2 b^{\prime}}$. That would induce terms of the type $\log \left(\frac{s_{2 b^{\prime}}}{s_{1 b^{\prime}}}\right)$ or $\log \left(\frac{s_{2 b^{\prime}}}{s_{1 b^{\prime}}+s_{2 b^{\prime}}}\right)$ in the impact factor $(2.24)$. Such terms would be harmless in NMRK, but would not yield the correct MRK limit (2.25).

Eq. (2.24) is the last ingredient which is necessary to evaluate the gluon-jet impact factor at NNLO accuracy in $\alpha_{S}$. It is also the first instance in which loop-level QCD amplitudes are evaluated in next-to-multi-Regge kinematics. ${ }^{7}$ This has led to the introduction of a specific Reggeisation ansatz for each colour-ordered amplitude (2.13). We

\footnotetext{
${ }^{7}$ Loop-level $\mathcal{N}=4 \mathrm{SYM}$ amplitudes have been considered in NMRK in order to compute the two-loop six-point remainder function [70, 71].
} 
expect that the same issue will arise in the evaluation of the one-loop corrections to the central-emission vertex for the production of two gluons or of a quark-antiquark pair along the gluon ladder, which is one of the building blocks, yet to be determined, of the BFKL kernel at NNLL accuracy.

\section{Acknowledgments}

We thank Einan Gardi for useful discussions and for a critical reading of the draft. VDD's work has been supported in part by the European Research Council (ERC) under grant agreement No 694712 (PertQCD). MC's work has been supported in part by the European Research Council under Advanced Investigator Grant ERC-AdG-885414.

\section{A Multiparton kinematics}

Without loss of generality, we consider the production of up to three partons of momenta $p_{1}$, $p_{2}$ and $p_{3}$, in the scattering between two partons of momenta $p_{a}$ and $p_{b}$. By convention, we consider the scattering in the unphysical region where all momenta are taken as outgoing, and then we analitically continue to the physical region where $p_{a}^{0}<0$ and $p_{b}^{0}<0$. Thus partons are incoming or outgoing depending on the sign of their energy. Since the helicity of a positive-energy (negative-energy) massless spinor has the same (opposite) sign as its chirality, the helicities assigned to the partons depend on whether they are incoming or outgoing. We label outgoing (positive-energy) particles with their helicity; if they are incoming the actual helicity and charge are reversed, e.g. an incoming left-handed parton is labelled as an outgoing right-handed anti-parton.

Using light-cone coordinates $p^{ \pm}=p_{0} \pm p_{z}$, and complex transverse coordinates $p_{\perp}=$ $p^{x}+i p^{y}$, with scalar product,

$$
2 p \cdot q=p^{+} q^{-}+p^{-} q^{+}-p_{\perp} q_{\perp}^{*}-p_{\perp}^{*} q_{\perp},
$$

the four-momenta are,

$$
\begin{aligned}
p_{a} & =\left(p_{a}^{+} / 2,0,0, p_{a}^{+} / 2\right) \equiv\left(p_{a}^{+}, 0 ; 0,0\right), \\
p_{b} & =\left(p_{b}^{-} / 2,0,0,-p_{b}^{-} / 2\right) \equiv\left(0, p_{b}^{-} ; 0,0\right), \\
p_{i} & =\left(\left(p_{i}^{+}+p_{i}^{-}\right) / 2, \operatorname{Re}\left[p_{i \perp}\right], \operatorname{Im}\left[p_{i \perp}\right],\left(p_{i}^{+}-p_{i}^{-}\right) / 2\right) \\
& \equiv\left(\left|p_{i \perp}\right| e^{y_{i}},\left|p_{i \perp}\right| e^{-y_{i}} ;\left|p_{i \perp}\right| \cos \phi_{i},\left|p_{i \perp}\right| \sin \phi_{i}\right),
\end{aligned}
$$

where $y$ is the rapidity, and $1 \leq i \leq 3$. The first notation in eq. (A.2) is the standard representation $p^{\mu}=\left(p^{0}, p^{x}, p^{y}, p^{z}\right)$, while in the second we have the + and - light-cone components on the left of the semicolon, and on the right the transverse components.

From the momentum conservation,

$$
\begin{gathered}
0=\sum_{i=1}^{3} p_{i \perp}, \\
p_{a}^{+}=-\sum_{i=1}^{3} p_{i}^{+},
\end{gathered}
$$




$$
p_{b}^{-}=-\sum_{i=1}^{3} p_{i}^{-}
$$

and using the scalar product eq. (A.1), the Mandelstam invariants may be written as,

$$
\begin{gathered}
s=2 p_{a} \cdot p_{b}=\sum_{i, j=1}^{3} p_{i}^{+} p_{j}^{-} \\
s_{a i}=2 p_{a} \cdot p_{i}=-\sum_{j=1}^{3} p_{i}^{-} p_{j}^{+} \\
s_{b i}=2 p_{b} \cdot p_{i}=-\sum_{j=1}^{3} p_{i}^{+} p_{j}^{-} .
\end{gathered}
$$

For the momenta (A.2), we use the spinor products [62]

$$
\begin{aligned}
\left\langle p_{i} p_{j}\right\rangle & =p_{i \perp} \sqrt{\frac{p_{j}^{+}}{p_{i}^{+}}}-p_{j \perp} \sqrt{\frac{p_{i}^{+}}{p_{j}^{+}}}, \\
\left\langle p_{a} p_{i}\right\rangle & =-i \sqrt{\frac{-p_{a}^{+}}{p_{i}^{+}}} p_{i \perp} \\
\left\langle p_{i} p_{b}\right\rangle & =i \sqrt{-p_{b}^{-} p_{i}^{+}} \\
\left\langle p_{a} p_{b}\right\rangle & =-\sqrt{p_{a}^{+} p_{b}^{-}}
\end{aligned}
$$

where we have used the mass-shell condition $\left|p_{i \perp}\right|^{2}=p_{i}^{+} p_{i}^{-}$.

\section{B Multi-Regge kinematics}

In the multi-Regge kinematics, we require that the gluons are strongly ordered in rapidity and have comparable transverse momentum,

$$
y_{1} \gg y_{2} \gg y_{3} ; \quad\left|p_{1 \perp}\right| \simeq\left|p_{2 \perp}\right| \simeq\left|p_{3 \perp}\right| .
$$

Momentum conservation (A.3) then becomes

$$
\begin{gathered}
0=\sum_{i=1}^{3} p_{i \perp}, \\
p_{a}^{+} \simeq-p_{1}^{+}, \\
p_{b}^{-} \simeq-p_{3}^{-} .
\end{gathered}
$$

The Mandelstam invariants (A.4) are reduced to,

$$
\begin{aligned}
s & =2 p_{a} \cdot p_{b} \simeq p_{1}^{+} p_{3}^{-}, \\
s_{a i} & =2 p_{a} \cdot p_{i} \simeq-p_{1}^{+} p_{i}^{-},
\end{aligned}
$$




$$
\begin{aligned}
& s_{b i}=2 p_{b} \cdot p_{i} \simeq-p_{i}^{+} p_{3}^{-}, \\
& s_{i j}=2 p_{i} \cdot p_{j} \simeq p_{i}^{+} p_{j}^{-} \quad \text { for } y_{i}>y_{j},
\end{aligned}
$$

to leading accuracy. The spinor products (A.5) become,

$$
\begin{aligned}
\left\langle p_{i} p_{j}\right\rangle & \simeq-\sqrt{\frac{p_{i}^{+}}{p_{j}^{+}}} p_{j \perp} \quad \text { for } y_{i}>y_{j} \\
\left\langle p_{a} p_{i}\right\rangle & \simeq-i \sqrt{\frac{p_{1}^{+}}{p_{i}^{+}}} p_{i \perp}, \\
\left\langle p_{i} p_{b}\right\rangle & \simeq i \sqrt{p_{i}^{+} p_{3}^{-}} \\
\left\langle p_{a} p_{b}\right\rangle & \simeq-\sqrt{p_{1}^{+} p_{3}^{-}} .
\end{aligned}
$$

\section{Next-to-multi-Regge kinematics}

We consider the production of three partons of momenta $p_{1}, p_{2}, p_{3}$, with partons 1 and 2 in the forward-rapidity region of parton $p_{a}$,

$$
y_{1} \simeq y_{2} \gg y_{3} ; \quad\left|p_{1 \perp}\right| \simeq\left|p_{2 \perp}\right| \simeq\left|p_{3 \perp}\right| .
$$

Momentum conservation (A.3) becomes

$$
\begin{aligned}
0 & =\sum_{i=1}^{3} p_{i \perp}, \\
p_{a}^{+} & \simeq-\left(p_{1}^{+}+p_{2}^{+}\right), \\
p_{b}^{-} & \simeq-p_{3}^{-} .
\end{aligned}
$$

The Mandelstam invariants (A.4) become

$$
\begin{array}{rlr}
s & \simeq\left(p_{1}^{+}+p_{2}^{+}\right) p_{3}^{-} & \\
s_{a 3} & \simeq-\left(p_{1}^{+}+p_{2}^{+}\right) p_{3}^{-} & \\
s_{a k} & \simeq-\left(p_{1}^{+}+p_{2}^{+}\right) p_{k}^{-} & \\
s_{b k} & \simeq-p_{k}^{+} p_{3}^{-} & k=1,2 \\
s_{b 3} & \simeq-p_{3}^{+} p_{3}^{-}=-p_{3 \perp}^{*} p_{3 \perp} & \\
s_{k 3} & \simeq p_{k}^{+} p_{3}^{-} & k=1,2 \\
s_{12} & =p_{1}^{+} p_{2}^{-}+p_{1}^{-} p_{2}^{+}-p_{1 \perp}^{*} p_{2 \perp}-p_{2 \perp}^{*} p_{1 \perp} .
\end{array}
$$

The spinor products (A.5) become

$$
\begin{aligned}
& \left\langle p_{a} p_{b}\right\rangle \simeq-\sqrt{\left(p_{1}^{+}+p_{2}^{+}\right) p_{3}^{-}} \\
& \left\langle p_{a} p_{3}\right\rangle=-i \sqrt{\frac{-p_{a}^{+}}{p_{3}^{+}}} p_{3 \perp} \simeq i \frac{p_{3 \perp}}{\left|p_{3 \perp}\right|}\left\langle p_{a} p_{b}\right\rangle,
\end{aligned}
$$




$$
\begin{array}{ll}
\left\langle p_{a} p_{k}\right\rangle=-i \sqrt{\frac{-p_{a}^{+}}{p_{k}^{+}}} p_{k \perp} \simeq-i \sqrt{\frac{p_{1}^{+}+p_{2}^{+}}{p_{k}^{+}}} p_{k \perp}, & k=1,2 \\
\left\langle p_{k} p_{b}\right\rangle=i \sqrt{-p_{b}^{-} p_{k}^{+}} \simeq i \sqrt{p_{k}^{+} p_{3}^{-}}, \quad k=1,2 \\
\left\langle p_{3} p_{b}\right\rangle=i \sqrt{-p_{b}^{-} p_{3}^{+}} \simeq i\left|p_{3 \perp}\right|, \\
\left\langle p_{k} p_{3}\right\rangle=p_{k \perp} \sqrt{\frac{p_{3}^{+}}{p_{k}^{+}}}-p_{3 \perp} \sqrt{\frac{p_{k}^{+}}{p_{3}^{+}}} \simeq-p_{3 \perp} \sqrt{\frac{p_{k}^{+}}{p_{3}^{+}}}, \quad k=1,2 \\
\left\langle p_{1} p_{2}\right\rangle=p_{1 \perp} \sqrt{\frac{p_{2}^{+}}{p_{1}^{+}}}-p_{2 \perp} \sqrt{\frac{p_{1}^{+}}{p_{2}^{+}}} .
\end{array}
$$

\section{Anomalous dimensions}

The perturbative expansion of the cusp anomalous dimension [72, 73], divided by the relevant quadratic Casimir factor $C_{i}$, is

$$
\gamma_{K}\left(\alpha_{S}\right)=\sum_{L=1}^{\infty} \gamma_{K}^{(L)}\left(\frac{\alpha_{s}}{\pi}\right)^{L}
$$

with

$$
\gamma_{K}^{(1)}=2 .
$$

The perturbative expansion of the collinear anomalous dimension is

$$
\gamma_{i}\left(\alpha_{S}\right)=\sum_{L=1}^{\infty} \gamma_{i}^{(L)}\left(\frac{\alpha_{s}}{\pi}\right)^{L}, \quad i=q, g
$$

with coefficients,

$$
\gamma_{g}^{(1)}=-\frac{\beta_{0}}{4}, \quad \gamma_{q}^{(1)}=-\frac{3}{4} C_{F},
$$

where $\beta_{0}$ is the coefficient of the beta function,

$$
\beta_{0}=\frac{11 N_{c}-2 N_{F}}{3},
$$

and

$$
C_{F}=\frac{N_{c}^{2}-1}{2 N_{c}}
$$

Note that, as customary in the literature, the expansion in eqs. (D.1) and (D.3) is in $\alpha_{s} / \pi$, while the impact factor (1.12) and the Regge trajectory (1.11) are expanded in $\alpha_{s} / 4 \pi$.

\section{E One-loop five-gluon colour-ordered amplitudes}

In this appendix, we report in the notation of ref. [43] the one-loop five-gluon colour-ordered amplitudes, which were computed in ref. [65]. The one-loop five-gluon colour-ordered 
amplitudes in eq. (2.23) can be written as [65]

$$
\begin{aligned}
m_{5: 1}^{[1]} & =\kappa_{\Gamma} m_{5 g}^{(0)}\left[V^{g}+4 V^{f}+V^{s}+4 G^{f}+G^{s}\right] \\
m_{5: 1}^{[1 / 2]} & =-\kappa_{\Gamma} m_{5 g}^{(0)}\left[V^{f}+V^{s}+G^{f}+G^{s}\right],
\end{aligned}
$$

with $\kappa_{\Gamma}$ as in eq. (1.8), and where the superscripts $g, f, s$ label contributions from an $N=4$ supersymmetric multiplet, an $N=1$ chiral multiplet, and a complex scalar, respectively. As done in ref. [43], in eq. (E.1) we have factored out the tree five-gluon colour-ordered amplitude.

For the one-loop impact factor $A^{g g(1)}(2.15)$, we need the one-loop five-gluon colourordered amplitudes only in the helicity configurations which are nonzero at tree level. We write the functions for the $(1,2,3,4,5)$ colour order for the two relevant helicity configurations below. The function obtained from the $N=4$ multiplet is the same for both helicity configurations [65],

$$
V^{g}=-\frac{1}{\epsilon^{2}} \sum_{j=1}^{5}\left(\frac{\mu^{2}}{-s_{j, j+1}}\right)^{\epsilon}+\sum_{j=1}^{5} \log \left(\frac{-s_{j, j+1}}{-s_{j+1, j+2}}\right) \log \left(\frac{-s_{j+2, j-2}}{-s_{j-2, j-1}}\right)+\frac{5}{6} \pi^{2}-\frac{\delta_{R}}{3} .
$$

The other functions depend on the helicity configuration. We define

$$
I_{i j k l}=[i j]\langle j k\rangle[k l]\langle l i\rangle .
$$

For the $\left(1^{-}, 2^{-}, 3^{+}, 4^{+}, 5^{+}\right)$helicity configuration we have [65],

$$
\begin{aligned}
V^{f}= & -\frac{5}{2 \epsilon}-\frac{1}{2}\left[\log \left(\frac{\mu^{2}}{-s_{23}}\right)+\log \left(\frac{\mu^{2}}{-s_{51}}\right)\right]-2 \\
G^{f}= & \frac{I_{1234}+I_{1245}}{2 s_{12} s_{51}} L_{0}\left(\frac{-s_{23}}{-s_{51}}\right) \\
G^{s}= & -\frac{G^{f}}{3}+\frac{I_{1234} I_{1245}\left(I_{1234}+I_{1245}\right)}{3 s_{12}^{3} s_{51}^{3}} L_{2}\left(\frac{-s_{23}}{-s_{51}}\right) \\
& +\frac{I_{1235}^{2}}{3 s_{12}^{2} s_{23} s_{51}}\left(1-\frac{s_{35}}{s_{12}}\right)+\frac{I_{1234} I_{1245}}{6 s_{12}^{2} s_{23} s_{51}},
\end{aligned}
$$

while for the $\left(1^{-}, 2^{+}, 3^{-}, 4^{+}, 5^{+}\right)$helicity configuration, we have

$$
\begin{aligned}
V^{f}= & -\frac{5}{2 \epsilon}-\frac{1}{2}\left[\log \left(\frac{\mu^{2}}{-s_{34}}\right)+\log \left(\frac{\mu^{2}}{-s_{51}}\right)\right]-2 \\
G^{f}= & -\frac{I_{1325}+I_{1342}}{2 s_{13} s_{51}} L_{0}\left(\frac{-s_{34}}{-s_{51}}\right)+\frac{I_{1324} I_{1342}}{s_{13}^{2} s_{51}^{2}} L s_{1}\left(\frac{-s_{23}}{-s_{51}}, \frac{-s_{34}}{-s_{51}}\right) \\
& +\frac{I_{1325} I_{1352}}{s_{13}^{2} s_{34}^{2}} L s_{1}\left(\frac{-s_{12}}{-s_{34}}, \frac{-s_{51}}{-s_{34}}\right) \\
G^{s}= & -\frac{I_{1324}^{2} I_{1342}^{2}}{s_{13}^{4} s_{24}^{2} s_{51}^{2}}\left[2 L s_{1}\left(\frac{-s_{23}}{-s_{51}}, \frac{-s_{34}}{-s_{51}}\right)+L_{1}\left(\frac{-s_{23}}{-s_{51}}\right)+L_{1}\left(\frac{-s_{34}}{-s_{51}}\right)\right] \\
& -\frac{I_{1325}^{2} I_{1352}^{2}}{s_{13}^{4} s_{25}^{2} s_{34}^{2}}\left[2 L s_{1}\left(\frac{-s_{12}}{-s_{34}}, \frac{-s_{51}}{-s_{34}}\right)+L_{1}\left(\frac{-s_{12}}{-s_{34}}\right)+L_{1}\left(\frac{-s_{51}}{-s_{34}}\right)\right]
\end{aligned}
$$




$$
\begin{aligned}
& +\frac{2}{3} \frac{I_{1324}^{3} I_{1342}}{s_{13}^{4} s_{24} s_{51}^{3}} L_{2}\left(\frac{-s_{23}}{-s_{51}}\right)+\frac{2}{3} \frac{I_{1352}^{3} I_{1325}}{s_{13}^{4} s_{25} s_{34}^{3}} L_{2}\left(\frac{-s_{12}}{-s_{34}}\right) \\
& +\frac{1}{3 s_{51}^{3}} L_{2}\left(\frac{-s_{34}}{-s_{51}}\right)\left[-\frac{I_{1325} I_{1342}\left(I_{1325}+I_{1342}\right)}{s_{13}^{3}}+2 \frac{I_{1342}^{3} I_{1324}}{s_{13}^{4} s_{24}}+2 \frac{I_{1325}^{3} I_{1352}}{s_{13}^{4} s_{25}}\right] \\
& +\frac{I_{1325}+I_{1342}}{6 s_{13} s_{51}} L_{0}\left(\frac{-s_{34}}{-s_{51}}\right)+\frac{I_{1325}^{2} I_{1342}^{2}}{3 s_{13}^{4} s_{23} s_{51} s_{34} s_{12}} \\
& +\frac{I_{1324}^{2} I_{1342}^{2}}{3 s_{13}^{4} s_{23} s_{24} s_{34} s_{51}}+\frac{I_{1325}^{2} I_{1352}^{2}}{3 s_{13}^{4} s_{25} s_{12} s_{34} s_{51}}-\frac{I_{1342} I_{1325}}{6 s_{13}^{2} s_{34} s_{51}}
\end{aligned}
$$

with

$$
\begin{aligned}
L_{0}(x)= & \frac{\log (x)}{1-x} \\
L_{1}(x)= & \frac{\log (x)+1-x}{(1-x)^{2}} \\
L_{2}(x)= & \frac{1}{(1-x)^{3}}\left[\log (x)-\frac{x}{2}+\frac{1}{2 x}\right] \\
L s_{1}(x, y)= & \frac{1}{(1-x-y)^{2}}\left[\operatorname{Li}_{2}(1-x)+\operatorname{Li}_{2}(1-y)\right. \\
& \left.+\log (x) \log (y)+(1-x-y)\left[L_{0}(x)+L_{0}(y)\right]-\frac{\pi^{2}}{6}\right],
\end{aligned}
$$

where $\mathrm{Li}_{2}$ is the dilogarithm.

For both the helicity configurations above, the functions $V^{s}$ and $V^{f}$ are related by,

$$
V^{s}=-\frac{V^{f}}{3}+\frac{2}{9}
$$

In the expansion in $\epsilon$, eq. (E.2)-(E.7) are valid to $O\left(\epsilon^{0}\right)$. The colour-ordered amplitudes (E.1) defined in terms of eqs. (E.2)-(E.7) are $\overline{\mathrm{MS}}$ regulated.

The colour-weighted sum,

$$
m_{5: 1}=N_{c} m_{5: 1}^{[1]}+N_{f} m_{5: 1}^{[1 / 2]},
$$

can be written as the sum of a universal piece, which is the same for both helicity configurations, and a non-universal piece, which depends on the helicity configuration,

$$
m_{5: 1}=m_{5: 1}^{u}+m_{5: 1}^{n u}
$$

where, using eqs. (E.1), (E.2) and (E.7), we can write

$$
\begin{aligned}
& m_{5: 1}^{u}=\kappa_{\Gamma} m_{5 g}^{(0)} N_{c} V^{g}, \\
& m_{5: 1}^{n u}=\kappa_{\Gamma} m_{5 g}^{(0)}\left[\beta_{0} V^{f}+\left(4 N_{c}-N_{f}\right) G^{f}+\left(N_{c}-N_{f}\right)\left(G^{s}+\frac{2}{9}\right)\right],
\end{aligned}
$$

which is useful to single out the $\overline{\mathrm{MS}}$ ultraviolet counterterm. 


\section{F The one-loop five-gluon amplitude in NMRK}

We evaluate the colour-ordered amplitudes $m_{5: 1}^{[1]}$ and $m_{5: 1}^{[1 / 2]}$, appendix E, in the NMRK region of eq. (2.1) and appendix C. We use the Mandelstam invariants (C.3) and the prescription,

$$
\log (-x)=\log (|x|)-i \pi \Theta(x),
$$

in order to select the real part of the one-loop five-gluon amplitude. For the helicity configuration $\left(a^{-}, 1^{+}, 2^{+}, b^{+}, b^{-}\right)$, the real part of the $\overline{\mathrm{MS}}$-renormalised one-loop five-gluon amplitude is

$$
\begin{aligned}
& \frac{1}{\kappa_{\Gamma} m_{5 g}^{(0)}} \operatorname{Re}\left[m_{5 ; 1}\left(p_{a}^{-}, p_{1}^{+}, p_{2}^{+}, p_{b^{\prime}}^{+}, p_{b}^{-}\right)\right] \\
& =N_{c}\left\{-\frac{1}{\epsilon^{2}}\left[2\left(\frac{\mu^{2}}{\left|p_{1 \perp}\right|^{2}}\right)^{\epsilon}+\left(\frac{\mu^{2}}{\left|p_{2 \perp}\right|^{2}}\right)^{\epsilon}+2\left(\frac{\mu^{2}}{-t}\right)^{\epsilon}\right]\right. \\
& +\frac{2}{\epsilon}\left[\left(\frac{\mu^{2}}{\left|p_{1 \perp}\right|^{2}}\right)^{\epsilon} \log \left(\frac{s_{12}}{\left|p_{1 \perp}\right|\left|p_{2 \perp}\right|}\right)+\left(\frac{\mu^{2}}{-t}\right)^{\epsilon} \log \left(\frac{x_{2} s}{\sqrt{-t}\left|p_{2 \perp}\right|}\right)\right. \\
& \left.-\frac{1}{2}\left(\frac{\mu^{2}}{-t} \frac{\left|p_{2 \perp}\right|^{2}}{\left|p_{1 \perp}\right|^{2}}\left(\frac{p_{2}^{+}}{p_{1}^{+}} \frac{s_{12}}{\left|p_{2 \perp}\right|^{2}}\right)^{\frac{1}{2}}\right)^{\epsilon} \log \left(\frac{x_{1} x_{2} s_{12}}{\left|p_{2 \perp}\right|^{2}}\right)\right] \\
& \left.-\frac{1}{2} \log ^{2}\left(\frac{\left|p_{1 \perp}\right|^{2}}{-t}\right)-\frac{1}{2} \log \left(x_{1}\right) \log \left(x_{1} x_{2}\left(\frac{-t}{s_{12}}\right)^{3} \frac{-t}{\left|p_{2 \perp}\right|^{2}}\right)-\frac{64}{9}-\frac{\delta_{R}}{3}+\frac{4}{3} \pi^{2}\right\} \\
& -\frac{\beta_{0}}{2 \epsilon}\left[\left(\frac{x_{1} \mu^{2}}{\left|p_{1 \perp}\right|^{2}}\right)^{\epsilon}+\left(\frac{\mu^{2}}{-t}\right)^{\epsilon}\right]+\frac{10}{9} N_{f}+\frac{\beta_{0}}{2}\left(\frac{\left|p_{1 \perp}\right|^{2}}{x_{1}}-t+2 q_{\perp}^{*} p_{1 \perp}\right) \frac{L_{0}\left(\frac{\left|p_{1 \perp}\right|^{2}}{-x_{1} t}\right)}{t} \\
& +\frac{N_{C}-N_{f}}{3}\left\{\frac{p_{2}^{+}}{p_{1}^{+}}\left[\left|p_{1 \perp}\right|^{2} q_{\perp}^{*} p_{2 \perp}^{2}-2\left|p_{1 \perp}\right|^{2}\left|p_{2 \perp}\right|^{2} p_{1 \perp} q_{\perp}^{*}+\frac{p_{2}^{+}}{p_{1}^{+}}\left|p_{1 \perp}\right|^{4} p_{2 \perp} q_{\perp}^{*}\right]\right. \\
& \left.-\left|p_{2 \perp}\right|^{2}\left[2\left|p_{1 \perp}\right|^{2}(-t)+\left(2\left|p_{2 \perp}\right|^{2}-\left|p_{1 \perp}\right|^{2}+t\right) p_{1 \perp} q_{\perp}^{*}\right]\right\} \frac{L_{2}\left(\frac{\left|p_{1 \perp}\right|^{2}}{-x_{1} t}\right)}{t^{3}} \\
& +\frac{N_{C}-N_{f}}{6}\left[\frac{x_{2} p_{2 \perp} q_{\perp}^{*}}{-t}+\frac{2 x_{1} x_{2} q_{\perp}^{*} p_{1 \perp}^{2}}{-t\left|p_{1 \perp}\right|^{2}}-\frac{x_{1}\left|p_{2 \perp}\right|^{2} p_{1 \perp} q_{\perp}^{*}}{-t\left|p_{1 \perp}\right|^{2}}\right]-\frac{3}{2} \frac{\beta_{0}}{\epsilon},
\end{aligned}
$$

where the last term is the $\overline{\mathrm{MS}}$ ultraviolet counterterm, where $q_{\perp}=-\left(p_{1 \perp}+p_{2 \perp}\right)$, with $t=-\left|q_{\perp}\right|^{2}$, and where it is understood that we must use

$$
\begin{aligned}
& 2 \operatorname{Re}\left(q_{\perp}^{*} p_{1 \perp}\right)=t+\left|p_{2 \perp}\right|^{2}-\left|p_{1 \perp}\right|^{2}, \\
& 2 \operatorname{Re}\left(q_{\perp}^{*} p_{2 \perp}\right)=t+\left|p_{1 \perp}\right|^{2}-\left|p_{2 \perp}\right|^{2} .
\end{aligned}
$$

In the MRK, $p_{1}^{+} \gg p_{2}^{+}$, the quantities that follow become,

$$
\begin{aligned}
x_{1} & \rightarrow 1 \\
x_{2} & \rightarrow \frac{p_{2}^{+}}{p_{1}^{+}} \\
s_{12} & \rightarrow \frac{p_{1}^{+}}{p_{2}^{+}}\left|p_{2 \perp}\right|^{2}
\end{aligned}
$$




$$
\begin{aligned}
\log \left(\frac{s_{12}}{\left|p_{1 \perp}\right|\left|p_{2 \perp}\right|}\right) & \rightarrow \log \left(\frac{s_{12}}{\sqrt{-t_{1}}\left|p_{2 \perp}\right|}\right) \\
\log \left(\frac{x_{2} s}{\sqrt{-t}\left|p_{2 \perp}\right|}\right) & \rightarrow \log \left(\frac{s_{23}}{\sqrt{-t_{2}}\left|p_{2 \perp}\right|}\right),
\end{aligned}
$$

with $t_{1}=-\left|p_{1 \perp}\right|^{2}$ and $t_{2}=-\left|p_{b^{\prime} \perp}\right|^{2}$. In particular, the logarithms in the last two lines of eq. (F.4), which occur on the second line of the right-hand side of eq. (F.2) become the two rapidity intervals of a five-gluon amplitude in MRK, and eq. (F.2) is reduced to the one-loop five-gluon amplitude in MRK [43].

\section{G Coefficients of the one-loop two-quark three-gluon amplitude in NMRK}

Here we display the real parts of the coefficients $\lambda_{1}$ and $\chi_{1}$, which are factored out in eqs. (2.42) and (2.48),

$$
\begin{aligned}
& \frac{1}{\kappa_{\Gamma}} \operatorname{Re}\left[\lambda_{1}\right]=-\frac{1}{\epsilon^{2}}\left[2\left(\frac{\mu^{2}}{\left|p_{1 \perp}\right|^{2}}\right)^{\epsilon}+\left(\frac{\mu^{2}}{\left|p_{2 \perp}\right|^{2}}\right)^{\epsilon}+\left(\frac{\mu^{2}}{-t}\right)^{\epsilon}\right] \\
& +\frac{2}{\epsilon}\left[\left(\frac{\mu^{2}}{\left|p_{1 \perp}\right|^{2}}\right)^{\epsilon} \log \left(\frac{s_{12}}{\left|p_{1 \perp}\right|\left|p_{2 \perp}\right|}\right)+\left(\frac{\mu^{2}}{-t}\right)^{\epsilon} \log \left(\frac{x_{2} s}{\sqrt{-t}\left|p_{2 \perp}\right|}\right)\right. \\
& \left.-\frac{1}{2}\left(\frac{\mu^{2}}{-t} \frac{\left|p_{2 \perp}\right|^{2}}{\left|p_{1 \perp}\right|^{2}}\left(\frac{p_{2}^{+}}{p_{1}^{+}} \frac{s_{12}}{\left|p_{2 \perp}\right|^{2}}\right)^{\frac{1}{2}}\right)^{\epsilon} \log \left(\frac{x_{1} x_{2} s_{12}}{\left|p_{2 \perp}\right|^{2}}\right)\right] \\
& -\left(\frac{\mu^{2}}{-t}\right)^{\epsilon}\left(\frac{3}{2 \epsilon}-\frac{83}{18}\right)-\left(\frac{\mu^{2}}{\left|p_{1 \perp}\right|^{2}}\right)^{\epsilon} \frac{11}{3 \epsilon}-\frac{11}{2 \epsilon} \\
& -\frac{1}{2} \log ^{2}\left(\frac{\left|p_{1 \perp}\right|^{2}}{-t}\right)-\frac{1}{2} \log \left(x_{1}\right) \log \left(x_{1} x_{2}\left(\frac{-t}{s_{12}}\right)^{3} \frac{-t}{\left|p_{2 \perp}\right|^{2}}\right) \\
& -\frac{11}{6} \log \left(x_{1}\right)+\frac{4}{3} \pi^{2}-\frac{64}{9}+\frac{11}{6}\left(\frac{\left|p_{1 \perp}\right|^{2}}{x_{1}}-t+2 q_{\perp}^{*} p_{1 \perp}\right) \frac{L_{0}\left(\frac{\left|p_{1 \perp}\right|^{2}}{-x_{1} t}\right)}{t} \\
& +\frac{1}{3}\left\{\frac{p_{2}^{+}}{p_{1}^{+}}\left[\left|p_{1 \perp}\right|^{2} q_{\perp}^{*} p_{2 \perp}^{2}-2\left|p_{1 \perp}\right|^{2}\left|p_{2 \perp}\right|^{2} p_{1 \perp} q_{\perp}^{*}+\frac{p_{2}^{+}}{p_{1}^{+}}\left|p_{1 \perp}\right|^{4} p_{2 \perp} q_{\perp}^{*}\right]\right. \\
& \left.-\left|p_{2 \perp}\right|^{2}\left[2\left|p_{1 \perp}\right|^{2}(-t)+\left(2\left|p_{2 \perp}\right|^{2}-\left|p_{1 \perp}\right|^{2}+t\right) p_{1 \perp} q_{\perp}^{*}\right]\right\} \frac{L_{2}\left(\frac{\left|p_{1 \perp}\right|^{2}}{-x_{1} t}\right)}{t^{3}} \\
& +\frac{1}{6}\left[\frac{x_{2} p_{2 \perp} q_{\perp}^{*}}{-t}+\frac{2 x_{1} x_{2} q_{\perp}^{*} p_{1 \perp}^{2}}{-t\left|p_{1 \perp}\right|^{2}}-\frac{x_{1}\left|p_{2 \perp}\right|^{2} p_{1 \perp} q_{\perp}^{*}}{-t\left|p_{1 \perp}\right|^{2}}\right],
\end{aligned}
$$

and $\lambda_{2}$ is obtained from $\lambda_{1}$ by swapping the labels 1 and 2 ,

$$
\begin{gathered}
\lambda_{2}=\lambda_{1}(1 \leftrightarrow 2) . \\
\frac{1}{\kappa_{\Gamma}} \operatorname{Re}\left[\chi_{1}\right]=\frac{1}{\epsilon}+\frac{2}{3 \epsilon}\left(\frac{\mu^{2}}{\left|p_{1 \perp}\right|^{2}}\right)^{\epsilon}-\frac{10}{9}\left(\frac{\mu^{2}}{-t}\right)^{\epsilon}+\frac{1}{3} \log \left(x_{1}\right)+\frac{10}{9} \\
-\frac{1}{3}\left(\frac{\left|p_{1 \perp}\right|^{2}}{x_{1}}-t+2 q_{\perp}^{*} p_{1 \perp}\right) \frac{L_{0}\left(\frac{\left|p_{1 \perp}\right|^{2}}{-x_{1} t}\right)}{t}
\end{gathered}
$$




$$
\begin{aligned}
& -\frac{1}{3}\left\{\frac{p_{2}^{+}}{p_{1}^{+}}\left[\left|p_{1 \perp}\right|^{2} q_{\perp}^{* 2} p_{2 \perp}^{2}-2\left|p_{1 \perp}\right|^{2}\left|p_{2 \perp}\right|^{2} p_{1 \perp} q_{\perp}^{*}+\frac{p_{2}^{+}}{p_{1}^{+}}\left|p_{1 \perp}\right|^{4} p_{2 \perp} q_{\perp}^{*}\right]\right. \\
& \left.-\left|p_{2 \perp}\right|^{2}\left[2\left|p_{1 \perp}\right|^{2}(-t)+\left(2\left|p_{2 \perp}\right|^{2}-\left|p_{1 \perp}\right|^{2}+t\right) p_{1 \perp} q_{\perp}^{*}\right]\right\} \frac{L_{2}\left(\frac{\left|p_{1 \perp}\right|^{2}}{-x_{1} t}\right)}{t^{3}} \\
& -\frac{1}{6}\left[\frac{x_{2} p_{2 \perp} q_{\perp}^{*}}{-t}+\frac{2 x_{1} x_{2} q_{\perp}^{*} p_{1 \perp}^{2}}{-t\left|p_{1 \perp}\right|^{2}}-\frac{x_{1}\left|p_{2 \perp}\right|^{2} p_{1 \perp} q_{\perp}^{*}}{-t\left|p_{1 \perp}\right|^{2}}\right]
\end{aligned}
$$

and $\chi_{2}$ is obtained from $\chi_{1}$ by swapping the labels 1 and 2 ,

$$
\chi_{2}=\chi_{1}(1 \leftrightarrow 2),
$$

where it is understood that in order to obtain the real part of eqs. (G.1)-(G.4), we must use eq. (F.3).

Open Access. This article is distributed under the terms of the Creative Commons Attribution License (CC-BY 4.0), which permits any use, distribution and reproduction in any medium, provided the original author(s) and source are credited.

\section{References}

[1] L.N. Lipatov, Reggeization of the vector meson and the vacuum singularity in nonabelian gauge theories, Sov. J. Nucl. Phys. 23 (1976) 338 [Yad. Fiz. 23 (1976) 642] [InSPIRE].

[2] L.J. Dixon, C. Duhr and J. Pennington, Single-valued harmonic polylogarithms and the multi-Regge limit, JHEP 10 (2012) 074 [arXiv:1207.0186] [INSPIRE].

[3] V. Del Duca, L.J. Dixon, C. Duhr and J. Pennington, The BFKL equation, Mueller-Navelet jets and single-valued harmonic polylogarithms, JHEP 02 (2014) 086 [arXiv:1309.6647] [INSPIRE].

[4] V. Del Duca et al., Multi-Regge kinematics and the moduli space of Riemann spheres with marked points, JHEP 08 (2016) 152 [arXiv: 1606.08807] [INSPIRE].

[5] V. Del Duca, C. Duhr, R. Marzucca and B. Verbeek, The analytic structure and the transcendental weight of the BFKL ladder at NLL accuracy, JHEP 10 (2017) 001 [arXiv: 1705.10163] [INSPIRE].

[6] V. Del Duca et al., All-order amplitudes at any multiplicity in the multi-Regge limit, Phys. Rev. Lett. 124 (2020) 161602 [arXiv: 1912.00188] [INSPIRE].

[7] S. Caron-Huot, E. Gardi, J. Reichel and L. Vernazza, Two-parton scattering amplitudes in the Regge limit to high loop orders, JHEP 08 (2020) 116 [arXiv:2006.01267] [INSPIRE].

[8] E.A. Kuraev, L.N. Lipatov and V.S. Fadin, Multi-Reggeon processes in the Yang-Mills theory, Sov. Phys. JETP 44 (1976) 443 [Zh. Eksp. Teor. Fiz. 71 (1976) 840] [INSPIRE].

[9] Z. Bern, J.J.M. Carrasco and H. Johansson, New relations for gauge-theory amplitudes, Phys. Rev. D 78 (2008) 085011 [arXiv: 0805.3993] [InSPIRE].

[10] V. Del Duca, Equivalence of the Parke-Taylor and the Fadin-Kuraev-Lipatov amplitudes in the high-energy limit, Phys. Rev. D 52 (1995) 1527 [hep-ph/9503340] [INSPIRE].

[11] I.I. Balitsky, L.N. Lipatov and V.S. Fadin, Regge processes in non-Abelian gauge theories (in Russian), ??. 
[12] V.S. Fadin, E.A. Kuraev and L.N. Lipatov, On the Pomeranchuk Singularity in asymptotically free theories, Phys. Lett. B 60 (1975) 50 [InSPIRE].

[13] E.A. Kuraev, L.N. Lipatov and V.S. Fadin, The Pomeranchuk singularity in nonabelian gauge theories, Sov. Phys. JETP 45 (1977) 199 [Zh. Eksp. Teor. Fiz. 72 (1977) 377] [InSPIRE].

[14] I.I. Balitsky and L.N. Lipatov, The Pomeranchuk singularity in quantum chromodynamics, Sov. J. Nucl. Phys. 28 (1978) 822 [ Yad. Fiz. 28 (1978) 1597] [INSPIRE].

[15] A.H. Mueller and H. Navelet, An inclusive minijet cross-section and the bare pomeron in QCD, Nucl. Phys. B 282 (1987) 727 [InSPIRE].

[16] V. Del Duca and C.R. Schmidt, Dijet production at large rapidity intervals, Phys. Rev. D 49 (1994) 4510 [hep-ph/9311290] [INSPIRE].

[17] W.J. Stirling, Production of jet pairs at large relative rapidity in hadron hadron collisions as a probe of the perturbative Pomeron, Nucl. Phys. B 423 (1994) 56 [hep-ph/9401266] [INSPIRE].

[18] J.R. Andersen, V. Del Duca, S. Frixione, C.R. Schmidt and W.J. Stirling, Mueller-Navelet jets at hadron colliders, JHEP 02 (2001) 007 [hep-ph/0101180] [INSPIRE].

[19] J.R. Andersen, V. Del Duca and C.D. White, Higgs boson production in association with multiple hard jets, JHEP 02 (2009) 015 [arXiv:0808.3696] [INSPIRE].

[20] J.R. Andersen and J.M. Smillie, Constructing all-order corrections to multi-jet rates, JHEP 01 (2010) 039 [arXiv:0908.2786] [inSPIRE].

[21] J.R. Andersen and J.M. Smillie, Multiple jets at the LHC with high energy jets, JHEP 06 (2011) 010 [arXiv:1101.5394] [INSPIRE].

[22] V.S. Fadin and L.N. Lipatov, Radiative corrections to QCD scattering amplitudes in a multi-Regge kinematics, Nucl. Phys. B 406 (1993) 259 [INSPIRE].

[23] V.S. Fadin, M.I. Kotsky and R. Fiore, Gluon Reggeization in QCD in the next-to-leading order, Phys. Lett. B 359 (1995) 181 [inSPIRE].

[24] V.S. Fadin, R. Fiore and M.I. Kotsky, Gluon Regge trajectory in the two loop approximation, Phys. Lett. B 387 (1996) 593 [hep-ph/9605357] [INSPIRE].

[25] V.S. Fadin, R. Fiore and A. Quartarolo, Reggeization of quark quark scattering amplitude in QCD, Phys. Rev. D 53 (1996) 2729 [hep-ph/9506432] [INSPIRE].

[26] J. Blumlein, V. Ravindran and W.L. van Neerven, On the gluon Regge trajectory in $O\left(\alpha_{S}^{2}\right)$, Phys. Rev. D 58 (1998) 091502 [hep-ph/9806357] [INSPIRE].

[27] V. Del Duca and E.W.N. Glover, The high-energy limit of QCD at two loops, JHEP 10 (2001) 035 [hep-ph/0109028] [inSPIRE].

[28] V.S. Fadin and R. Fiore, Quark contribution to the gluon-gluon-reggeon vertex in QCD, Phys. Lett. B 294 (1992) 286 [INSPIRE].

[29] V.S. Fadin, R. Fiore and A. Quartarolo, Radiative corrections to quark quark reggeon vertex in QCD, Phys. Rev. D 50 (1994) 2265 [hep-ph/9310252] [INSPIRE].

[30] V. Del Duca and C.R. Schmidt, Virtual next-to-leading corrections to the impact factors in the high-energy limit, Phys. Rev. D 57 (1998) 4069 [hep-ph/9711309] [INSPIRE].

[31] Z. Bern, V. Del Duca and C.R. Schmidt, The infrared behavior of one loop gluon amplitudes at next-to-next-to-leading order, Phys. Lett. B 445 (1998) 168 [hep-ph/9810409] [INSPIRE]. 
[32] V.S. Fadin, R. Fiore, M.G. Kozlov and A.V. Reznichenko, Proof of the multi-Regge form of QCD amplitudes with gluon exchanges in the NLA, Phys. Lett. B 639 (2006) 74 [hep-ph/0602006] [INSPIRE].

[33] V.S. Fadin, M.G. Kozlov and A.V. Reznichenko, Gluon reggeization in Yang-Mills theories, Phys. Rev. D 92 (2015) 085044 [arXiv:1507.00823] [INSPIRE].

[34] V.S. Fadin and L.N. Lipatov, BFKL Pomeron in the next-to-leading approximation, Phys. Lett. B 429 (1998) 127 [hep-ph/9802290] [INSPIRE].

[35] M. Ciafaloni and G. Camici, Energy scale(s) and next-to-leading BFKL equation, Phys. Lett. B 430 (1998) 349 [hep-ph/9803389] [INSPIRE].

[36] V.S. Fadin and L.N. Lipatov, High-energy production of gluons in a quasimulti-Regge kinematics, JETP Lett. 49 (1989) 352 [Yad. Fiz. 50 (1989) 1141] [INSPIRE].

[37] V. Del Duca, Real next-to-leading corrections to the multi-gluon amplitudes in the helicity formalism, Phys. Rev. D 54 (1996) 989 [hep-ph/9601211] [INSPIRE].

[38] V.S. Fadin and L.N. Lipatov, Next-to-leading corrections to the BFKL equation from the gluon and quark production, Nucl. Phys. B 477 (1996) 767 [hep-ph/9602287] [INSPIRE].

[39] V. Del Duca, Quark-anti-quark contribution to the multi-gluon amplitudes in the helicity formalism, Phys. Rev. D 54 (1996) 4474 [hep-ph/9604250] [INSPIRE].

[40] V. Del Duca, Next-to-leading corrections to the BFKL equation, Frascati Phys. Ser. 5 (1996) 463 [hep-ph/9605404] [INSPIRE].

[41] V.S. Fadin, R. Fiore and A. Quartarolo, Quark contribution to the reggeon - reggeon - gluon vertex in QCD, Phys. Rev. D 50 (1994) 5893 [hep-th/9405127] [INSPIRE].

[42] V.S. Fadin, R. Fiore and M.I. Kotsky, Gribov's theorem on soft emission and the reggeon-reggeon-gluon vertex at small transverse momentum, Phys. Lett. B 389 (1996) 737 [hep-ph/9608229] [INSPIRE].

[43] V. Del Duca and C.R. Schmidt, Virtual next-to-leading corrections to the Lipatov vertex, Phys. Rev. D 59 (1999) 074004 [hep-ph/9810215] [INSPIRE].

[44] D. Colferai, F. Schwennsen, L. Szymanowski and S. Wallon, Mueller Navelet jets at LHCComplete NLL BFKL calculation, JHEP 12 (2010) 026 [arXiv:1002.1365] [INSPIRE].

[45] B. Ducloue, L. Szymanowski and S. Wallon, Confronting Mueller-Navelet jets in NLL BFKL with LHC experiments at $7 \mathrm{TeV}$, JHEP 05 (2013) 096 [arXiv:1302.7012] [INSPIRE].

[46] B. Ducloué, L. Szymanowski and S. Wallon, Evidence for high-energy resummation effects in Mueller-Navelet jets at the LHC, Phys. Rev. Lett. 112 (2014) 082003 [arXiv:1309.3229] [INSPIRE].

[47] J. Bartels, D. Colferai and G.P. Vacca, The NLO jet vertex for Mueller-Navelet and forward jets: the quark part, Eur. Phys. J. C 24 (2002) 83 [hep-ph/0112283] [INSPIRE].

[48] J. Bartels, D. Colferai and G.P. Vacca, The NLO jet vertex for Mueller-Navelet and forward jets: the gluon part, Eur. Phys. J. C 29 (2003) 235 [hep-ph/0206290] [InSPIRE].

[49] S. Caron-Huot, E. Gardi and L. Vernazza, Two-parton scattering in the high-energy limit, JHEP 06 (2017) 016 [arXiv: 1701.05241] [INSPIRE].

[50] S. Caron-Huot, When does the gluon reggeize?, JHEP 05 (2015) 093 [arXiv:1309.6521] [INSPIRE]. 
[51] S. Caron-Huot, E. Gardi, J. Reichel and L. Vernazza, Infrared singularities of QCD scattering amplitudes in the Regge limit to all orders, JHEP 03 (2018) 098 [arXiv: 1711.04850] [INSPIRE].

[52] V.S. Fadin, Particularities of the NNLLA BFKL, AIP Conf. Proc. 1819 (2017) 060003 [arXiv: 1612.04481] [INSPIRE].

[53] V.S. Fadin and L.N. Lipatov, Reggeon cuts in QCD amplitudes with negative signature, Eur. Phys. J. C 78 (2018) 439 [arXiv:1712.09805] [INSPIRE].

[54] G. Falcioni, E. Gardi, C. Milloy and L. Vernazza, Climbing three-Reggeon ladders: four-loop amplitudes in the high-energy limit in full colour, arXiv:2012.00613 [INSPIRE].

[55] V. Del Duca, C. Duhr, E. Gardi, L. Magnea and C.D. White, An infrared approach to Reggeization, Phys. Rev. D 85 (2012) 071104 [arXiv:1108.5947] [InSPIRE].

[56] V. Del Duca, C. Duhr, E. Gardi, L. Magnea and C.D. White, The infrared structure of gauge theory amplitudes in the high-energy limit, JHEP 12 (2011) 021 [arXiv:1109.3581] [INSPIRE].

[57] V. Del Duca, G. Falcioni, L. Magnea and L. Vernazza, High-energy QCD amplitudes at two loops and beyond, Phys. Lett. B 732 (2014) 233 [arXiv:1311.0304] [INSPIRE].

[58] V. Del Duca, G. Falcioni, L. Magnea and L. Vernazza, Analyzing high-energy factorization beyond next-to-leading logarithmic accuracy, JHEP 02 (2015) 029 [arXiv:1409.8330] [INSPIRE].

[59] I.A. Korchemskaya and G.P. Korchemsky, Evolution equation for gluon Regge trajectory, Phys. Lett. B 387 (1996) 346 [hep-ph/9607229] [INSPIRE].

[60] J.M. Henn and B. Mistlberger, Four-gluon scattering at three loops, infrared structure, and the Regge limit, Phys. Rev. Lett. 117 (2016) 171601 [arXiv:1608.00850] [INSPIRE].

[61] L.N. Lipatov, Integrability of scattering amplitudes in $N=4$ SUSY, J. Phys. A 42 (2009) 304020 [arXiv: 0902 .1444] [INSPIRE].

[62] V. Del Duca, A. Frizzo and F. Maltoni, Factorization of tree QCD amplitudes in the high-energy limit and in the collinear limit, Nucl. Phys. B 568 (2000) 211 [hep-ph/9909464] [INSPIRE].

[63] E.N. Antonov, L.N. Lipatov, E.A. Kuraev and I.O. Cherednikov, Feynman rules for effective Regge action, Nucl. Phys. B 721 (2005) 111 [hep-ph/0411185] [INSPIRE].

[64] C. Duhr, New techniques in QCD, Ph.D. thesis, Université Catholique de Louvain, Belgium (2009).

[65] Z. Bern, L.J. Dixon and D.A. Kosower, One loop corrections to five gluon amplitudes, Phys. Rev. Lett. 70 (1993) 2677 [hep-ph/9302280] [INSPIRE].

[66] Z. Bern, L.J. Dixon and D.A. Kosower, One loop corrections to two quark three gluon amplitudes, Nucl. Phys. B 437 (1995) 259 [hep-ph/9409393] [INSPIRE].

[67] V. Del Duca, Iterating QCD scattering amplitudes in the high-energy limit, JHEP 02 (2018) 112 [arXiv: 1712.07030] [INSPIRE].

[68] S. Catani, M.H. Seymour and Z. Trócsányi, Regularization scheme independence and unitarity in QCD cross-sections, Phys. Rev. D 55 (1997) 6819 [hep-ph/9610553] [INSPIRE].

[69] V. Del Duca, L.J. Dixon and F. Maltoni, New color decompositions for gauge amplitudes at tree and loop level, Nucl. Phys. B 571 (2000) 51 [hep-ph/9910563] [INSPIRE]. 
[70] V. Del Duca, C. Duhr and V.A. Smirnov, An analytic result for the two-loop hexagon Wilson loop in $N=4$ SYM, JHEP 03 (2010) 099 [arXiv:0911.5332] [INSPIRE].

[71] V. Del Duca, C. Duhr and V.A. Smirnov, The two-loop hexagon Wilson loop in $N=4 S Y M$, JHEP 05 (2010) 084 [arXiv: 1003.1702] [inSPIRE].

[72] G.P. Korchemsky and A.V. Radyushkin, Loop space formalism and renormalization group for the infrared asymptotics of QCD, Phys. Lett. B 171 (1986) 459 [INSPIRE].

[73] S. Moch, J.A.M. Vermaseren and A. Vogt, The Three loop splitting functions in QCD: the nonsinglet case, Nucl. Phys. B 688 (2004) 101 [hep-ph/0403192] [INSPIRE]. 